Appl. Set-Valued Anal. Optim. 4 (2022), No. 1, pp. 1-26

Available online at http://asvao.biemdas.com

https://doi.org/10.23952/asvao.4.2022.1.01

\title{
KARUSH-KUHN-TUCKER OPTIMALITY CONDITIONS AND DUALITY FOR NONSMOOTH MULTIOBJECTIVE SEMI-INFINITE PROGRAMMING PROBLEMS WITH VANISHING CONSTRAINTS
}

\author{
LE THANH TUNG \\ Department of Mathematics, College of Natural Sciences, Can Tho University, Can Tho 900000, Vietnam
}

\begin{abstract}
This paper considers the nonsmooth multiobjective semi-infinite programming problems with vanishing constraints. Using Clarke subdifferentials, we first obtain both necessary and sufficient Karush-Kuhn-Tucker optimality conditions for nonsmooth multiobjective semi-infinite programming problems with vanishing constraints. Then, the duality relations of types of Wolfe and Mond-Weir dual problems are explored under convexity assumptions.

Keywords. Clarke subdifferentials; Constraint qualifications; Karush-Kuhn-Tucker optimality conditions; Mond-Weir and Wolfe duality; Multiobjective semi-infinite programming problems.
\end{abstract}

\section{INTRODUCTION}

The mathematical programming problems with vanishing constraints, proposed in $[1,2]$, are applied in reformulating many problems from structural topology optimization in certain engineering applications. Several constraint qualifications and the corresponding Karush-KuhnTucker (KKT) necessary optimality conditions were given in [3, 4]. The paper [5] investigated the concepts of stationary points of mathematical programming problems with vanishing constraints under a topological point of view on the critical point theory. In [6], strong KKT necessary optimality conditions for multiobjective mathematical programming problems with vanishing constraints were explored. The papers [7,8] studied some constraint qualifications in terms of Clarke subdifferentials and applied them in establishing the KKT optimality conditions for nonsmooth mathematical programming problems. Some results on the duality for mathematical programming problems with vanishing constraints were discussed in $[9,10]$. In addition, the semi-infinite programming problems, the optimization with an infinite number of constraints, have been recently studied by many authors; see, e.g., $[11,12,13,14,15,16,17,18,19,20]$ and the references therein. Employing the Clarke subdifferentials, the KKT sufficient optimality conditions for nonsmooth semi-infinite programming problems with vanishing constraints were investigated in [21]. The papers [22, 23, 24] investigated KKT necessary and sufficient optimality conditions and duality for smooth semi-infinite programming problems with vanishing constraints. However, KKT necessary optimality conditions for nonsmooth semi-infinite programming problems with vanishing constraints have not yet considered in [21]. Moreover,

E-mail address: lttung@ctu.edu.vn.

Received September 20, 2021; Accepted October 23, 2021.

(C)2022 Applied Set-Valued Analysis and Optimization 
to the best of our knowledge, there is no paper dealing with duality for nonsmooth semi-infinite programming problems with vanishing constraints.

Motivated by the above observations, in this paper, we establish Karush-Kuhn-Tucker optimality conditions and investigate duality problems for the nonsmooth multiobjective semiinfinite programming problems with vanishing constraints. The paper is organized as follows. The basic concepts and some preliminaries are recalled in Section 2. The KKT necessary and sufficient optimality conditions for the semi-infinite programming problems with vanishing constraints in terms of Clarke subdifferentials are discussed in Section 3. Section 4, the last section, is devoted to delving into Mond-Weir and Wolfe dual problems of the nonsmooth multiobjective semi-infinite programming problems with vanishing constraints.

\section{PRELIMINARIES}

In this paper, the notation $\langle\cdot, \cdot\rangle$ is used to denote the inner product in the Euclidean space $\mathbb{R}^{n}$. By $B(x, \delta)$, we designate the open ball centered at $x$ with radius $\delta>0$. For $A \subseteq \mathbb{R}^{n}, \operatorname{int} A, \operatorname{cl} A$, $\partial \mathrm{A}, \operatorname{span} A$, and $\operatorname{co} A$ stand for its interior, closure, boundary, linear hull, and convex hull of $A$, respectively. The cone and the convex cone (containing the origin) generated by $A$ are denoted resp by cone $A$, and $\operatorname{pos} A$. It should be mentioned that, for the given sets $A_{1}, A_{2}$ in $\mathbb{R}^{n}$,

$$
\operatorname{span}\left(A_{1} \cup A_{2}\right)=\operatorname{span} A_{1}+\operatorname{span} A_{2} \text { and } \operatorname{pos}\left(A_{1} \cup A_{2}\right)=\operatorname{pos} A_{1}+\operatorname{pos} A_{2} .
$$

The negative polar cone, the strictly negative polar cone, and the orthogonal complement of $A$ are defined respectively by

$$
\begin{aligned}
& A^{-}:=\left\{x^{*} \in \mathbb{R}^{n} \mid\left\langle x^{*}, x\right\rangle \leq 0, \forall x \in A\right\}, \\
& A^{s}:=\left\{x^{*} \in \mathbb{R}^{n} \mid\left\langle x^{*}, x\right\rangle<0, \forall x \in A\right\},
\end{aligned}
$$

and

$$
A^{\perp}:=\left\{x^{*} \in \mathbb{R}^{n} \mid\left\langle x^{*}, x\right\rangle=0, \forall x \in A\right\} .
$$

It is easy to check that $A^{s} \subset A^{-}$and if $A^{s} \neq \emptyset$, then $\mathrm{cl} A^{s}=A^{-}$. Moreover, the bipolar theorem (see, e.g., [25]) states that $A^{--}=\operatorname{cl} \operatorname{pos} A$. For a given nonempty subset $A$ of $\mathbb{R}^{n}$, the contingent cone [25] of $A$ at $\bar{x} \in \operatorname{cl} A$ is

$$
\mathscr{T}(A, \bar{x}):=\left\{x \in \mathbb{R}^{n} \mid \exists \tau_{k} \downarrow 0, \exists x_{k} \rightarrow x, \forall k \in \mathbb{N}, \bar{x}+\tau_{k} x_{k} \in A\right\} .
$$

Note that if $A$ is a convex set, then $\mathscr{T}(A, \bar{x})=\operatorname{clcone}(A-\bar{x})$. If $\left\langle x^{*}, x\right\rangle \geq 0\left(\left\langle x^{*}, x\right\rangle=0\right)$ for all $x^{*} \in A^{*}$, where $A^{*}$ is a subset of the dual space of $\mathbb{R}^{n}$, we write $\left\langle A^{*}, x\right\rangle \geq 0\left(\left\langle A^{*}, x\right\rangle \geq 0\right.$, resp.). The notion $o\left(\tau^{k}\right)$, for $\tau>0$ and $k \in \mathbb{N}$, indicates a moving point such that $o\left(\tau^{k}\right) / \tau^{k} \rightarrow 0$ as $\tau \rightarrow 0^{+}$. The cardinality of the index set $I$ is denoted by $|I|$. For an index subset $I \subset\{1, \ldots, n\}$, $x_{I}=0\left(x_{I} \geq 0\right)$ stands for $x_{i}=0\left(x_{i} \geq 0\right.$, respectively $)$ for all $i \in I$.

For the set-valued map $F: \mathbb{R}^{n} \rightrightarrows \mathbb{R}^{m}$, the domain and graph of $F$ are defined respectively by

$$
\operatorname{dom} F:=\left\{x \in \mathbb{R}^{n} \mid F(x) \neq \emptyset\right\}, \operatorname{gr} F:=\left\{(x, y) \in \mathbb{R}^{n} \times \mathbb{R}^{m} \mid y \in F(x)\right\} .
$$

The system of the neighborhoods of a set $A \subset \mathbb{R}^{n}$, denoted by $\mathscr{U}(A)$, is defined by

$$
\mathscr{U}(A):=\left\{U \subset \mathbb{R}^{n} \mid U \text { is open and } U \supset A\right\} .
$$


When $A$ is a compact set, the subsets $\mathbf{B}(A, \eta)=\left\{x \in \mathbb{R}^{n} \mid d(x, A)<\eta\right\}$ form a system of the neighborhoods of $A$. In the case that $A=\bar{x}, \mathscr{U}(\bar{x})$ is the system of the neighborhoods of $\bar{x}$. The Painlevé-Kuratowski (sequential) outer (or upper) limit is defined by

$$
\underset{x_{x \rightarrow \bar{x}}}{\operatorname{Limsup}} F(x)=\left\{y \in \mathbb{R}^{m} \mid \exists x_{n} \in \operatorname{dom} F: x_{k} \rightarrow \bar{x}, \exists y_{k} \in F\left(x_{k}\right), y_{k} \rightarrow y\right\},
$$

where $x \stackrel{F}{\rightarrow} \bar{x}$ means that $x_{k} \in \operatorname{dom} F$ and $x_{k} \rightarrow \bar{x}$.

Definition 2.1. $[16,25,26]$ Let $F: \mathbb{R}^{n} \rightrightarrows \mathbb{R}^{m}$ and $\bar{x} \in \operatorname{dom} F$.

(i) $F$ is upper semicontinuous at $\bar{x}$ if, for any $U \in \mathscr{U}(F(\bar{x}))$, there exists $\delta>0$ such that $F(B(\bar{x}, \delta)) \subset U$. If $F(\bar{x})$ is a compact set, $F$ is upper semicontinuous at $\bar{x}$ if, for any $\eta>0$, there exists $\delta>0$ such that $F(B(\bar{x}, \delta)) \subset \mathbf{B}(F(\bar{x}), \eta)$.

(ii) $F$ is closed at $\bar{x}$ if, for any $\left(x_{k}, y_{k}\right) \in \operatorname{gr} F$ and $\left(x_{k}, y_{k}\right) \rightarrow(\bar{x}, y) \in \mathbb{R}^{n} \times \mathbb{R}^{m}$, one has $y \in F(\bar{x})$.

It is easy to justify that if $F$ is upper semicontinuous at $\bar{x}$ and $F(\bar{x})$ is compact then $F$ is closed at $\bar{x}$ and $\operatorname{Limsup} F(x)=F(\bar{x})$.

$$
x \rightarrow \bar{x}
$$

Definition 2.2. [27] Let $\bar{x} \in \mathbb{R}^{n}$ and $\phi: \mathbb{R}^{n} \rightarrow \mathbb{R}$ be a locally Lipschitz function. The Clarke directional derivative of $\phi: \mathbb{R}^{n} \rightarrow \mathbb{R}$ at $\bar{x}$ in direction $u$ is defined by

$$
\phi^{o}(\bar{x}, u):=\limsup _{\tau \downarrow 0, x \rightarrow \bar{x}} \frac{\phi(x+\tau u)-\phi(x)}{\tau} .
$$

The Clarke subdifferential of $\phi$ at $\bar{x}$ is

$$
\partial^{C} \phi(\bar{x}):=\left\{x^{*} \in \mathbb{R}^{n} \mid\left\langle x^{*}, d\right\rangle \leq \phi^{o}(\bar{x}, d), \forall d \in \mathbb{R}^{n}\right\} .
$$

We recall the following properties from [27].

Lemma 2.1. Let $\phi, \psi$ be the functions from $\mathbb{R}^{n}$ to $\mathbb{R}$, which are Lipschitz near $\bar{x}$. Then, the following assertions hold:

(i) the function $v \rightarrow \phi^{o}(\bar{x}, v)$ is finite, positively homogenous, subadditive on $\mathbb{R}^{n}, \phi^{o}(\bar{x}, 0)=$ $0, \phi^{o}(\bar{x}, v)=\max _{x^{*} \in \partial^{C} \phi(\bar{x})}\left\langle x^{*}, v\right\rangle$ and $\partial\left(\phi^{o}(\bar{x},).\right)(0)=\partial^{C} \phi(\bar{x})$, where $\partial$ denotes the subdifferential in sense of convex analysis;

(ii) $\partial^{C} \phi(\bar{x})$ is a nonempty, convex and compact subset of $\mathbb{R}^{n}$;

(iii) $\partial^{C}(\lambda \phi)(\bar{x})=\lambda \partial^{C} \phi(\bar{x}), \forall \lambda \in \mathbb{R}$ and $\partial^{C}(\phi+\psi)(\bar{x}) \subseteq \partial^{C} \phi(\bar{x})+\partial^{C} \psi(\bar{x})$;

(iv) if $\phi$ is convex on $\mathbb{R}^{n}$ then $\partial^{C} \phi(\bar{x})=\partial \phi(\bar{x})$. If $\phi$ is continously differentiable at $\bar{x}$, then $\partial^{C} \phi(\bar{x})=\{\nabla \phi(\bar{x})\}$

(v) $\partial^{C} \phi(\bar{x})=\operatorname{co}\left\{x^{*} \in \mathbb{R}^{n} \mid \exists x_{k} \rightarrow \bar{x}, x_{k} \notin S \cup \Omega_{\phi}, \nabla f\left(x_{k}\right) \rightarrow x^{*}\right\}$, where $S$ is any set of Lebesgue measure 0 in $\mathbb{R}^{n}$ and $\Omega_{\phi}$ is the set of points at which a given function $\phi$ fails to be differentiable;

(vi) if $\phi$ is locally Lipschitz on $\mathbb{R}^{n}$, then $x \rightrightarrows \partial^{C} \phi(x)$ is an upper semicontinuous set-valued function;

(vii) if $\phi$ is locally Lipschitz on an open set containing $[x, y]$, then $\phi(x)-\phi(y)=\left\langle x^{*}, y-x\right\rangle$, for some $c \in[x, y)$ and $x^{*} \in \partial^{C} \phi(c)$. 
Example 2.1. (see [27, p. 64]) Let $f, h: \mathbb{R}^{2} \rightarrow \mathbb{R}$ be defined by $f(x)=\max \left\{h(x), x_{2}-x_{1}\right\}$, where $h(x)=\min \left\{x_{1},-x_{2}\right\}$. Then,

$$
h(x)= \begin{cases}-x_{2}, & \text { if } x_{2} \geq-x_{1} \\ x_{1}, & \text { if } x_{2}<-x_{1}\end{cases}
$$

and

$$
f(x)= \begin{cases}h(x), & \text { if } h(x) \geq x_{2}-x_{1}, \\ x_{2}-x_{1}, & \text { if } h(x)<x_{2}-x_{1}\end{cases}
$$

Since $h(x) \geq x_{2}-x_{1} \Leftrightarrow\left[\begin{array}{ll}-x_{2} \geq x_{2}-x_{1}, & \text { and } x_{2} \geq-x_{1}, \\ x_{1} \geq x_{2}-x_{1}, & \text { and } x_{2}<-x_{1} .\end{array} \Leftrightarrow\left[\begin{array}{ll}x_{2} \leq \frac{1}{2} x_{1} & \text { and } x_{2} \geq-x_{1}, \\ x_{2} \leq 2 x_{1} & \text { and } x_{2}<-x_{1},\end{array}\right.\right.$ $f(x)= \begin{cases}-x_{2}, & \text { if } x \in C_{1}:=\left\{x \in \mathbb{R}^{2} \mid x_{2} \leq \frac{1}{2} x_{1}, x_{2} \geq-x_{1}\right\}, \\ x_{1}, & \text { if } x \in C_{2}:=\left\{x \in \mathbb{R}^{2} \mid x_{2} \leq 2 x_{1}, x_{2}<-x_{1}\right\}, \\ x_{2}-x_{1}, & \text { if } x \in C_{3}:=\left\{x \in \mathbb{R}^{2} \mid x_{2}>\frac{1}{2} x_{1}, x_{2}>-x_{1}\right\} \cup\left\{x \in \mathbb{R}^{2} \mid x_{2}>2 x_{1}, x_{2} \leq-x_{1}\right\} .\end{cases}$
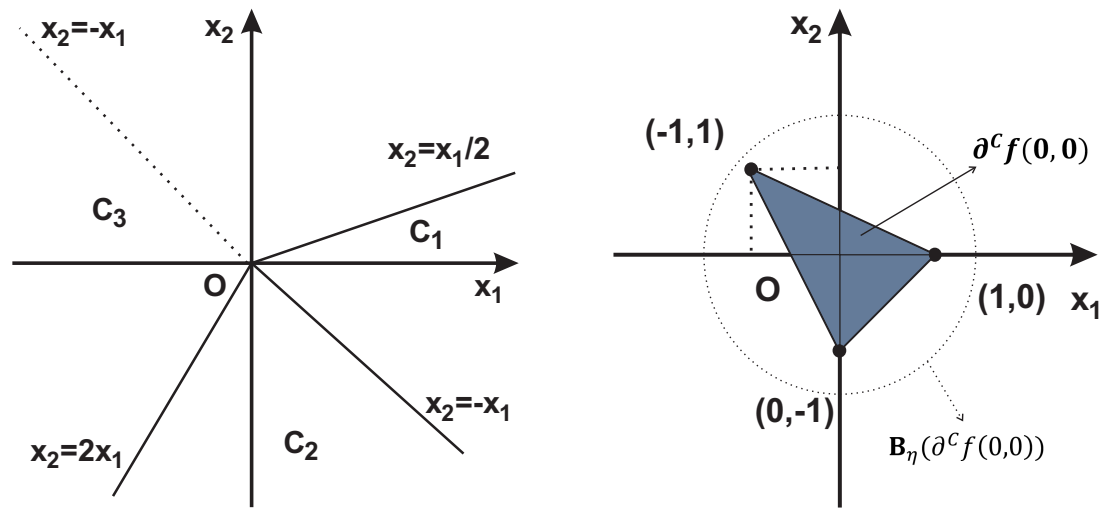

Figure 1. The graph of $C_{1}, C_{2}, C_{3}$ Figure 2. The graph of $\partial^{C} f(0,0)$

Since, for $x=(1,0) \in C_{1}, x^{\prime}=(0,-1) \in C_{2}$, and $\lambda=\frac{2}{3} \in[0,1]$,

$$
\lambda x+(1-\lambda) x^{\prime}=\left(\frac{2}{3},-\frac{1}{3}\right) \in C_{1},
$$

and

$$
f\left(\lambda x+(1-\lambda) x^{\prime}\right)=\frac{1}{3}>\frac{2}{3} \cdot 0+\frac{1}{3} \cdot 0=\lambda f(x)+(1-\lambda) f\left(x^{\prime}\right),
$$

$f$ is not convex on $\mathbb{R}^{2}$. However, we can check that $f$ is Lipschitz on $\mathbb{R}^{2}$. Since $\partial C_{1}, \partial C_{2}, \partial C_{2}$, and $\partial C_{1} \cup \partial C_{2} \cup \partial C_{1}$ are the sets of Lebesgue measure 0 in $\mathbb{R}^{2}$, one seesthat

$$
\partial^{C} f(x)= \begin{cases}\{(0,-1)\}, & \text { if } x \in C_{1} \backslash \partial C_{1}, \\ \operatorname{co}\{(0,-1),(1,0)\}, & \text { if } x \in\left(\partial C_{1} \cap \partial C_{2}\right) \backslash\{(0,0)\}, \\ \{(1,0)\}, & \text { if } x \in C_{2} \backslash \partial C_{2}, \\ \operatorname{co}\{(1,0),(-1,1)\}, & \text { if } x \in\left(\partial C_{2} \cap \partial C_{3}\right) \backslash\{(0,0)\}, \\ \{(-1,1)\}, & \text { if } x \in C_{3} \backslash \partial C_{3}, \\ \operatorname{co}\{(-1,1),(0,-1)\}, & \text { if } x \in\left(\partial C_{3} \cap \partial C_{1}\right) \backslash\{(0,0)\}, \\ \operatorname{co}\{(0,-1),(1,0),(-1,1)\}, & \text { if } x=(0,0) .\end{cases}
$$

Furthermore, we can verify that the set-valued map $x \rightrightarrows \partial^{C} f(x)$ is upper semicontinuous on $\mathbb{R}^{2}$. For instance, for any $\eta>0$, there exists $\delta=\eta$ such that $\partial^{C} f\left(B_{\delta}(0,0)\right) \subseteq \partial^{C} f((0,0)) \subseteq$ $\mathbf{B}_{\eta}\left(\partial^{C} f((0,0))\right)$, which implies that $\partial^{C} f$ is upper semicontinuous at $\bar{x}=(0,0)$. 
In view of [21], we consider the following multiobjective semi-infinite programming with vanishing constraints $(\mathrm{P})$ :

$$
\begin{array}{cl}
\mathbb{R}_{+}^{m}-\min & f(x)=\left(f_{1}(x), \ldots, f_{m}(x)\right) \\
\text { s.t. } & g_{t}(x) \leq 0, t \in T, \\
& h_{i}(x)=0, i=1, \ldots, q, \\
& H_{i}(x) \geq 0, i=1, \ldots, l, \\
& G_{i}(x) H_{i}(x) \leq 0, i=1, \ldots, l,
\end{array}
$$

where $f_{i}(i=1, \ldots, m), g_{t}(t \in T), h_{i}(i=1, \ldots, q)$, and $G_{i}, H_{i}(i=1, \ldots, l)$ are Lipschitzian functions from $\mathbb{R}^{n}$ to $\mathbb{R}$. The index set $T$ is an arbitrary nonempty set, not necessary finite. Let us denote $I:=\{1, \ldots, m\}, I_{h}:=\{1, \ldots, q\}$ and $I_{l}:=\{1, \ldots, l\}$. The feasible solution set of $(\mathrm{P})$ is

$$
\Omega:=\left\{x \in \mathbb{R}^{n} \mid g_{t}(x) \leq 0(t \in T), h_{i}(x)=0\left(i \in I_{h}\right), H_{i}(x) \geq 0, G_{i}(x) H_{i}(x) \leq 0\left(i \in I_{l}\right)\right\} .
$$

Recall some types of efficient solutions (see, e.g., [28]) of the multiobjective semi-infinite programming with vanishing constraints as follows.

Definition 2.3. Let $\bar{x} \in \Omega$.

(i) $\bar{x}$ is a locally (Pareto) efficient solution of (P), denoted by $\bar{x} \in \operatorname{loc} E(\mathrm{P})$, if there exists a neighborhood $U \in \mathscr{U}(\bar{x})$ such that there is no $x \in \Omega \cap U$ satisfying

$$
\begin{cases}f_{i}(x) \leq f_{i}(\bar{x}), & \forall i \in I, \\ f_{i_{0}}(x)<f_{i_{0}}(\bar{x}), & \text { for at least one } i_{0} \in I .\end{cases}
$$

(ii) $\bar{x}$ is a locally weakly (Pareto) efficient solution of (P), denoted by $\bar{x} \in \operatorname{locWE}(\mathrm{P})$, if there exists a neighborhood $U \in \mathscr{U}(\bar{x})$ such that there is no $x \in \Omega \cap U$ fulfilling

$$
f_{i}(x)<f_{i}(\bar{x}), \forall i \in I \text {. }
$$

If $U=\mathbb{R}^{n}$, the word "locally" is omitted. In this case, the efficient solution sets / the weakly efficient solution sets is denoted by $E(P) / W E(P)$. It is straightforward that $E(P) \subset W E(P)$.

The notation $\mathbb{R}_{+}^{|T|}$ signifies the collection of all the functions $\lambda: T \rightarrow \mathbb{R}$ taking values $\lambda_{t}$ 's positive only at finitely many points of $T$, and equal to zero at the other points. For a given $\bar{x} \in \Omega, I_{g}(\bar{x}):=\left\{t \in T \mid g_{t}(\bar{x})=0\right\}$ indicates the index set of all active constraints at $\bar{x}$. The set of active constraint multipliers at $\bar{x} \in \Omega$ is

$$
\Lambda(\bar{x}):=\left\{\lambda \in \mathbb{R}_{+}^{|T|} \mid \lambda_{t} g_{t}(\bar{x})=0, \forall t \in T\right\} .
$$

Notice that $\lambda \in \Lambda(\bar{x})$ if there exists a finite index set $J \subset I_{g}(\bar{x})$ such that $\lambda_{t}>0$ for all $t \in J$ and $\lambda_{t}=0$ for all $t \in T \backslash J$. For each $\bar{x} \in \Omega$, define

$$
\begin{aligned}
I_{+}(\bar{x}):=\left\{i \in I_{l} \mid H_{i}(\bar{x})>0\right\}, I_{0}(\bar{x}):=\left\{i \in I_{l} \mid H_{i}(\bar{x})=0\right\}, \\
I_{+0}(\bar{x}):=\left\{i \in I_{l} \mid H_{i}(\bar{x})>0, G_{i}(\bar{x})=0\right\} \\
I_{+-}(\bar{x}):=\left\{i \in I_{l} \mid H_{i}(\bar{x})>0, G_{i}(\bar{x})<0\right\} \\
I_{0+}(\bar{x}):=\left\{i \in I_{l} \mid H_{i}(\bar{x})=0, G_{i}(\bar{x})>0\right\} \\
I_{00}(\bar{x}):=\left\{i \in I_{l} \mid H_{i}(\bar{x})=0, G_{i}(\bar{x})=0\right\}
\end{aligned}
$$

and

$$
I_{0-}(\bar{x}):=\left\{i \in I_{l} \mid H_{i}(\bar{x})=0, G_{i}(\bar{x})<0\right\} .
$$

Definition 2.4. Let $\bar{x} \in \Omega$. 
(i) The point $\bar{x}$ is called a strong stationary point of (P) iff there exists $\left(\alpha, \lambda^{g}, \lambda^{h}, \lambda^{G}, \lambda^{H}\right) \in$ $\mathbb{R}_{+}^{m} \times \Lambda(\bar{x}) \times \mathbb{R}^{q} \times \mathbb{R}^{l} \times \mathbb{R}^{l}$ with $\sum_{i \in I} \alpha_{i}=1, \lambda_{I_{+}(\bar{x})}^{H}=0, \lambda_{I_{00}(\bar{x}) \cup I_{0-}(\bar{x})}^{H} \geq 0$,

$$
\lambda_{I_{+-}(\bar{x}) \cup I_{0+}(\bar{x}) \cup I_{00}(\bar{x}) \cup I_{0-}(\bar{x})}^{G}=0,
$$

and $\lambda_{I_{+0}(\bar{x})}^{G} \geq 0$ such that

$0 \in \sum_{i \in I} \alpha_{i} \partial^{C} f_{i}(\bar{x})+\sum_{t \in T} \lambda_{t}^{g} \partial^{C} g_{t}(\bar{x})+\sum_{i \in I_{h}} \lambda_{i}^{h} \partial^{C} h_{i}(\bar{x})-\sum_{i \in I_{l}} \lambda_{i}^{H} \partial^{C} H_{i}(\bar{x})+\sum_{i \in I_{l}} \lambda_{i}^{G} \partial^{C} G_{i}(\bar{x})$.

In the case that $\alpha_{i}>0, \forall i=1, \ldots, m, \bar{x}$ is called a proper strong stationary point of $(\mathrm{P})$ (see [21]).

(ii) The point $\bar{x}$ is said to be a VC-stationary point of (P) iff there exists $\left(\alpha, \lambda^{g}, \lambda^{h}, \lambda^{G}, \lambda^{H}\right) \in$ $\mathbb{R}_{+}^{m} \times \Lambda(\bar{x}) \times \mathbb{R}^{q} \times \mathbb{R}^{l} \times \mathbb{R}^{l}$ with $\sum_{i \in I} \alpha_{i}=1, \lambda_{I_{+}(\bar{x})}^{H}=0, \lambda_{I_{00}(\bar{x}) \cup I_{0-}(\bar{x})}^{H} \geq 0$,

$$
\lambda_{I_{+-}(\bar{x}) \cup I_{0+}(\bar{x}) \cup I_{0-}(\bar{x})}^{G}=0
$$

and $\lambda_{I_{+0}(\bar{x}) \cup I_{00}(\bar{x})} \geq 0$ satisfying

$$
0 \in \sum_{i \in I} \alpha_{i} \partial^{C} f_{i}(\bar{x})+\sum_{t \in T} \lambda_{t}^{g} \partial^{C} g_{t}(\bar{x})+\sum_{i \in I_{h}} \lambda_{i}^{h} \partial^{C} h_{i}(\bar{x})-\sum_{i \in I_{l}} \lambda_{i}^{H} \partial^{C} H_{i}(\bar{x})+\sum_{i \in I_{l}} \lambda_{i}^{G} \partial^{C} G_{i}(\bar{x}) .
$$

It is easy to see that if $\bar{x} \in \Omega$ is a strong stationary point of $(\mathrm{P})$, then $\bar{x}$ is a VC-stationary point of $(\mathrm{P})$.

For $\bar{x} \in \Omega$ and $\left(\lambda^{g}, \lambda^{h}, \lambda^{G}, \lambda^{H}\right) \in \mathbb{R}_{+}^{|T|} \times \mathbb{R}^{q} \times \mathbb{R}^{l} \times \mathbb{R}^{l}$, let us define

$$
\begin{gathered}
I_{g}^{+}(\bar{x}):=\left\{t \in I_{g}(\bar{x}) \mid \lambda_{t}^{g}>0\right\}, \\
I_{h}^{+}(\bar{x}):=\left\{i \in I_{h}(\bar{x}) \mid \lambda_{i}^{h}>0\right\}, I_{h}^{-}(\bar{x}):=\left\{i \in I_{h}(\bar{x}) \mid \lambda_{i}^{h}<0\right\}, \\
\hat{I}_{+}^{+}(\bar{x}):=\left\{i \in I_{+}(\bar{x}) \mid \lambda_{i}^{H}>0\right\}, \\
\hat{I}_{0}^{+}(\bar{x}):=\left\{i \in I_{0}(\bar{x}) \mid \lambda_{i}^{H}>0\right\}, \hat{I}_{0}^{-}(\bar{x}):=\left\{i \in I_{0}(\bar{x}) \mid \lambda_{i}^{H}<0\right\}, \\
\hat{I}_{0+}^{+}(\bar{x}):=\left\{i \in I_{0+}(\bar{x}) \mid \lambda_{i}^{H}>0\right\}, \hat{I}_{0+}^{-}(\bar{x}):=\left\{i \in I_{0+}(\bar{x}) \mid \lambda_{i}^{H}<0\right\}, \\
\hat{I}_{00}^{+}(\bar{x}):=\left\{i \in I_{00}(\bar{x}) \mid \lambda_{i}^{H}>0\right\}, \hat{I}_{00}^{-}(\bar{x}):=\left\{i \in I_{00}(\bar{x}) \mid \lambda_{i}^{H}<0\right\}, \\
\hat{I}_{0-}^{+}(\bar{x}):=\left\{i \in I_{0-}(\bar{x}) \mid \lambda_{i}^{H}>0\right\}, \\
I_{+0}^{+}(\bar{x}):=\left\{i \in I_{+0}(\bar{x}) \mid \lambda_{i}^{G}>0\right\}, I_{+0}^{-}(\bar{x}):=\left\{i \in I_{+0}(\bar{x}) \mid \lambda_{i}^{G}<0\right\}, \\
I_{+-}^{+}(\bar{x}):=\left\{i \in I_{+-}(\bar{x}) \mid \lambda_{i}^{G}>0\right\}, \\
I_{0+}^{+}(\bar{x}):=\left\{i \in I_{0+}(\bar{x}) \mid \lambda_{i}^{G}>0\right\}, I_{0+}^{-}(\bar{x}):=\left\{i \in I_{0+}(\bar{x}) \mid \lambda_{i}^{G}<0\right\}, \\
I_{00}^{+}(\bar{x}):=\left\{i \in I_{00}(\bar{x}) \mid \lambda_{i}^{G}>0\right\}, I_{00}^{-}(\bar{x}):=\left\{i \in I_{00}(\bar{x}) \mid \lambda_{i}^{G}<0\right\},
\end{gathered}
$$

and

$$
I_{0-}^{+}(\bar{x}):=\left\{i \in I_{0-}(\bar{x}) \mid \lambda_{i}^{G}>0\right\} .
$$

Definition 2.5. (see [29, 30]) Let $\phi: \mathbb{R}^{n} \rightarrow \mathbb{R}$ be a locally Lipschitz function, $\Omega \subset \mathbb{R}^{n}$, and $\bar{x} \in \Omega$.

(i) $\phi$ is said to be $\partial^{C}$-convex at $\bar{x}$ on $\Omega$ if, for all $x \in \Omega$,

$$
\phi(x)-\phi(\bar{x}) \geq\left\langle\partial^{C} \phi(\bar{x}), x-\bar{x}\right\rangle .
$$


(ii) $\phi$ is said to be strictly $\partial^{C}$-convex at $\bar{x}$ on $\Omega$ if, for all $x \in \Omega \backslash\{\bar{x}\}$,

$$
\phi(x)-\phi(\bar{x})>\left\langle\partial^{C} \phi(\bar{x}), x-\bar{x}\right\rangle .
$$

(iii) $\phi$ is termed $\partial^{C}$-pseudoconvex at $\bar{x}$ on $\Omega$ if, for any $x \in \Omega$,

$$
\phi(x)-\phi(\bar{x})<0 \Rightarrow\left\langle\partial^{C} \phi(\bar{x}), x-\bar{x}\right\rangle<0 .
$$

(iv) $\phi$ is declared strictly $\partial^{C}$-pseudoconvex at $\bar{x}$ on $\Omega$ if,for all $x \in \Omega \backslash\{\bar{x}\}$,

$$
\phi(x)-\phi(\bar{x}) \leq 0 \Rightarrow\left\langle\partial^{C} \phi(\bar{x}), x-\bar{x}\right\rangle<0 .
$$

(v) $\phi$ is said to be $\partial^{C}$-quasiconvex at $\bar{x}$ on $\Omega$ if, for any $x \in \Omega$,

$$
\phi(x)-\phi(\bar{x}) \leq 0 \Rightarrow\left\langle\partial^{C} \phi(\bar{x}), x-\bar{x}\right\rangle \leq 0 .
$$

Lemma 2.2. [31] Let $\left\{C_{t} \mid t \in \Gamma\right\}$ be an arbitrary collection of nonempty convex sets in $\mathbb{R}^{n}$, and $K=\operatorname{pos}\left(\cup_{t \in \Gamma} C_{t}\right)$. Then, every nonzero vector of $K$ can be expressed as a non-negative linear combination of $n$ or fewer linear independent vectors, each belonging to a different $C_{t}$.

Lemma 2.3. [32] Suppose that $S, T, P$ are arbitrary (possibly infinite) index sets, $a_{s}=a(s)=$ $\left(a_{1}(s), \ldots, a_{n}(s)\right)$ maps $S$ onto $\mathbb{R}^{n}$, and so do $a_{t}$ and $a_{p}$. Suppose that the set $\operatorname{co}\left\{a_{s}, s \in S\right\}+$ $\operatorname{pos}\left\{a_{t}, t \in T\right\}+\operatorname{span}\left\{a_{p}, p \in P\right\}$ is closed. Then the following statements are equivalent:

$$
\begin{gathered}
I: \quad\left\{\begin{array}{l}
\left\langle a_{s}, x\right\rangle<0, s \in S, S \neq \emptyset \\
\left\langle a_{t}, x\right\rangle \leq 0, t \in T \\
\left\langle a_{p}, x\right\rangle=0, p \in P
\end{array}\right. \\
I I: \quad 0 \in \operatorname{co}\left\{a_{s}, s \in S\right\}+\operatorname{pos}\left\{a_{t}, t \in T\right\}+\operatorname{span}\left\{a_{p}, p \in P\right\} .
\end{gathered}
$$

Lemma 2.4. [33] If $A$ is a nonempty compact subset of $\mathbb{R}^{n}$, then

(i) $\operatorname{co} A$ is a compact set;

(ii) if $0 \notin \operatorname{co} A$, then $\operatorname{pos} A$ is a closed cone.

\section{KARUSH-KUHN-TUCKER OPTIMALITY CONDITIONS}

In this section, we establish both KKT necessary and sufficient optimality conditions for the nonsmooth multiobjective semi-infinite programming with vanishing constraints. For the simplicity, we write the index set $I_{g}$ instead of $I_{g}(\bar{x})$. The other index sets are described similarly. Firstly, we present the following constraint qualifications, which are similar to Abadie constraint qualification in the literature:

(i) (ACQ) holds at $\bar{x} \in \Omega$ if

$$
\begin{aligned}
& \left(\bigcup_{t \in I_{g}} \partial^{C} g_{t}(\bar{x})\right)^{-} \cap\left(\bigcup_{i \in I_{h}} \partial^{C} h_{i}(\bar{x})\right)^{\perp} \cap\left(\bigcup_{i \in I_{0+}} \partial^{C} H_{i}(\bar{x})\right)^{\perp} \cap\left(\bigcup_{i \in I_{00} \cup I_{0-}}-\partial^{C} H_{i}(\bar{x})\right)^{-} \cap\left(\bigcup_{i \in I_{+0}} \partial^{C} G_{i}(\bar{x})\right)^{-} \\
& \subseteq \mathscr{T}(\Omega, \bar{x}),
\end{aligned}
$$

(ii)(VC-ACQ) holds at $\bar{x} \in \Omega$ if

$$
\begin{aligned}
& \left(\bigcup_{t \in I_{g}} \partial^{C} g_{t}(\bar{x})\right)^{-} \cap\left(\bigcup_{i \in I_{h}} \partial^{C} h_{i}(\bar{x})\right)^{\perp} \cap\left(\bigcup_{i \in I_{0+}} \partial^{C} H_{i}(\bar{x})\right)^{\perp} \cap\left(\bigcup_{i \in I_{00} \cup I_{0-}}-\partial^{C} H_{i}(\bar{x})\right)^{-} \cap\left(\bigcup_{i \in I_{+0} \cup I_{00}} \partial^{C} G_{i}(\bar{x})\right)^{-} \\
& \subseteq \mathscr{T}(\Omega, \bar{x}) .
\end{aligned}
$$


It is evident that (ACQ) implies (VC-ACQ). The following example illuminates that the reversal is not true in general.

Example 3.1. Let $m=n=2$ and $l=1$. Consider the following (P):

$$
\begin{array}{cc}
\mathbb{R}_{+}^{2}-\min & f(x)=\left(x_{1}^{2}+x_{2}^{2},\left|x_{1}\right|\right), \\
\text { s.t. } \quad & g_{t}(x)=-t x_{1} \leq 0, t \in T=\mathbb{N}, \\
& H_{1}(x)=\left(x_{1}^{3}+2 x_{2}\right) \geq 0, \\
& G_{1}(x) H_{1}(x)=\left|x_{1}\right|\left(x_{1}^{3}+2 x_{2}\right) \leq 0 .
\end{array}
$$

Then, $\Omega=\left\{x \in \mathbb{R}^{2} \mid x_{1}>0, x_{1}^{3}+2 x_{2}=0\right\} \cup\left\{x \in \mathbb{R}^{2} \mid x_{1}=0, x_{2} \geq 0\right\}$. For $\bar{x}=(0,0) \in \Omega$, direct calculations give that

$$
\begin{gathered}
\mathscr{T}(\Omega, \bar{x})=\left\{x \in \mathbb{R}^{2} \mid x_{1} \geq 0, x_{2}=0\right\} \cup\left\{x \in \mathbb{R}^{2} \mid x_{1}=0, x_{2} \geq 0\right\}, \\
I_{g}=\mathbb{N}, \partial^{C} g_{t}(\bar{x})=\{(-t, 0)\}, t \in T, \\
I_{+}=I_{0+}=I_{0-}=\emptyset, I_{00}=\{1\}, \partial^{C} G_{1}(\bar{x})=[-1,1] \times\{0\}, \partial^{C} H_{1}(\bar{x})=\{(0,2)\} \\
\left(\bigcup_{t \in I_{g}} \partial^{C} g_{t}(\bar{x})\right)^{-}=\left\{x \in \mathbb{R}^{2} \mid x_{1} \geq 0\right\} \\
\left(\bigcup_{i \in I_{00}}\left(-\partial^{C} H_{i}(\bar{x})\right)^{-}=\left(-\partial^{C} H_{1}(\bar{x})\right)^{-}=\left\{x \in \mathbb{R}^{2} \mid x_{2} \geq 0\right\}\right. \\
\left(\bigcup_{i \in I_{00}} \partial^{C} G_{i}(\bar{x})\right)^{-}=\left(\partial^{C} G_{1}(\bar{x})\right)^{-}=\left\{x \in \mathbb{R}^{2} \mid x_{1}=0\right\} \\
\left(\bigcup_{t \in I_{g}} \partial^{C} g_{t}(\bar{x})\right)^{-} \cap\left(\bigcup_{i \in I_{00}}\left(-\partial^{C} H_{i}(\bar{x})\right)\right)^{-}=\left\{x \in \mathbb{R}^{2} \mid x_{1} \geq 0, x_{2} \geq 0\right\}
\end{gathered}
$$

and

$$
\left(\bigcup_{t \in I_{g}} \partial^{C} g_{t}(\bar{x})\right)^{-} \cap\left(\bigcup_{i \in I_{00}}\left(-\partial^{C} H_{i}(\bar{x})\right)\right)^{-} \cap\left(\bigcup_{i \in I_{00}} \partial^{C} G_{i}(\bar{x})\right)^{-}=\left\{x \in \mathbb{R}^{2} \mid x_{1}=0, x_{2} \geq 0\right\} .
$$

Hence,

$$
\left(\bigcup_{t \in I_{g}} \partial^{C} g_{t}(\bar{x})\right)^{-} \cap\left(\bigcup_{i \in I_{00}}\left(-\partial^{C} H_{i}(\bar{x})\right)\right)^{-} \not \subset \mathscr{T}(\Omega, \bar{x})
$$

and

$$
\left(\bigcup_{t \in I_{g}} \partial^{C} g_{t}(\bar{x})\right)^{-} \cap\left(\bigcup_{i \in I_{00}}\left(-\partial^{C} H_{i}(\bar{x})\right)\right)^{-} \cap\left(\bigcup_{i \in I_{00}} \partial^{C} G_{i}(\bar{x})\right)^{-} \subset \mathscr{T}(\Omega, \bar{x}) .
$$

Thus, (ACQ) does not hold at $\bar{x}$ and (VC-ACQ) holds at $\bar{x}$.

Proposition 3.1. Let $\bar{x} \in \operatorname{loc} \mathrm{WE}(\mathrm{P})$.

(i) If (ACQ) holds at $\bar{x}$ and the set

$$
\begin{aligned}
\Delta:= & \operatorname{pos}\left(\bigcup_{t \in I_{g}} \partial^{C} g_{t}(\bar{x}) \cup \bigcup_{i \in I_{00} \cup I_{0-}}\left(-\partial^{C} H_{i}(\bar{x})\right) \cup \bigcup_{i \in I_{+0}} \partial^{C} G_{i}(\bar{x})\right) \\
& +\operatorname{span}\left(\bigcup_{i \in I_{h}} \partial^{C} h_{i}(\bar{x}) \cup \bigcup_{i \in I_{0+}} \partial^{C} H_{i}(\bar{x})\right)
\end{aligned}
$$

is closed, then $\bar{x}$ is a strong stationary point of $(P)$. 
(ii) If (VC-ACQ) holds at $\bar{x}$ and the set

$$
\begin{aligned}
\Delta_{1}:= & \operatorname{pos}\left(\bigcup_{t \in I_{g}} \partial^{C} g_{t}(\bar{x}) \cup \bigcup_{i \in I_{00} \cup I_{0-}}\left(-\partial^{C} H_{i}(\bar{x})\right) \cup \bigcup_{i \in I_{+0} \cup I_{00}} \partial^{C} G_{i}(\bar{x})\right) \\
& +\operatorname{span}\left(\bigcup_{i \in I_{h}} \partial^{C} h_{i}(\bar{x}) \cup \bigcup_{i \in I_{0+}} \partial^{C} H_{i}(\bar{x})\right)
\end{aligned}
$$

is closed, then $\bar{x}$ is a VC-stationary point of $(P)$.

Proof. Owing to the similarity, we only prove (ii). As $\bar{x} \in \operatorname{locWE}(\mathrm{P})$, there exists $U \in \mathscr{U}(\bar{x})$ such that there is no $x \in \Omega \cap U$ satisfying

$$
f_{i}(x)<f_{i}(\bar{x}), \quad \forall i \in I .
$$

First, we justify that

$$
\left(\bigcup_{i \in I} \partial^{C} f_{i}(\bar{x})\right)^{s} \cap \mathscr{T}(\Omega, \bar{x})=\emptyset
$$

There are two possibilities.

Case 1. $0 \in \partial^{C} f_{i_{0}}(\bar{x})$ for some $i_{0} \in I$. Then, $\left(\cup_{i \in I} \partial^{C} f_{i}(\bar{x})\right)^{s}=\emptyset$. Hence, (3.2) holds.

Case 2. $0 \notin \partial^{C} f_{i}(\bar{x})$ for all $i \in I$. Suppose to the contrary that there exists $d \in\left(\cup_{i \in I} \partial^{C} f_{i}(\bar{x})\right)^{s} \cap$ $\mathscr{T}(\Omega, \bar{x})$. As $d \in \mathscr{T}(\Omega, \bar{x})$, there exist $\tau_{k} \downarrow 0$ and $d_{k} \rightarrow d$ such that $\bar{x}+\tau_{k} d_{k} \in \Omega$ for all $k \in \mathbb{N}$. Since $d \in\left(\cup_{i \in I} \partial^{C} f_{i}(\bar{x})\right)^{s}$ and $0 \notin \partial^{C} f_{i}(\bar{x})$ for all $i \in I$, one has

$$
\left\langle\xi_{i}, d\right\rangle<0, \quad \forall \xi_{i} \in \partial^{C} f_{i}(\bar{x}), \forall i \in I .
$$

By the mean-value theorem in Lemma 2.2 (vii), for each $k \in \mathbb{N}$, there exists $u_{k}$ in the open segment $\left(\bar{x}, \bar{x}+\tau_{k}^{(1)} d_{k}^{(1)}\right)$, where $\tau_{k}^{(1)}:=\tau_{k}, d_{k}^{(1)}:=d_{k}$, and $\xi_{k}^{(1)} \in \partial^{C} f_{1}\left(u_{k}\right)$, such that

$$
f_{1}\left(\bar{x}+\tau_{k}^{(1)} d_{k}^{(1)}\right)-f_{1}(\bar{x})=\tau_{k}\left\langle\xi_{k}^{(1)}, d_{k}^{(1)}\right\rangle .
$$

Since $\partial^{C} f_{1}\left(u_{k}\right)$ are compact in $\mathbb{R}^{n}$ and $\left\{\xi_{k}^{(1)}\right\} \subset\left\{\partial^{C} f_{1}\left(u_{k}\right)\right\},\left\{\xi_{k}^{(1)}\right\}$ is bounded in $\mathbb{R}^{n}$. From the facts that $u_{k} \rightarrow \bar{x}$, the mapping $\partial^{C} f_{1}$ is upper semicontinuous at $\bar{x}$, and $\left\{\xi_{k}^{(1)}\right\}$ is bounded in $\mathbb{R}^{n}$, we deduce that there exists a subsequence of $\xi_{k}^{(1)}$, also denoted by $\xi_{k}^{(1)}$, such that $\xi_{k}^{(1)} \rightarrow$ $\xi_{1} \in \partial^{C} f_{1}(\bar{x})$. If follows from (3.3) that $\left\langle\xi_{1}, d\right\rangle<0$. We deduce from $\left\langle\xi_{k}^{(1)}, d_{k}^{(1)}\right\rangle \rightarrow\left\langle\xi_{1}, d\right\rangle<0$ and (3.4) that

$$
\frac{f_{1}\left(\bar{x}+\tau_{k}^{(1)} d_{k}^{(1)}\right)-f_{1}(\bar{x})}{\tau_{k}^{(1)}}=\left\langle\xi_{k}^{(1)}, d_{k}^{(1)}\right\rangle \rightarrow\left\langle\xi_{1}, d\right\rangle<0 .
$$

This leads that, for $k$ large enough,

$$
\frac{f_{1}\left(\bar{x}+\tau_{k}^{(1)} d_{k}^{(1)}\right)-f_{1}(\bar{x})}{\tau_{k}^{(1)}}<0,
$$

which together with $\tau_{k}^{(1)}>0\left(\tau_{k}^{(1)} \downarrow 0\right)$ implies that $f_{1}\left(\bar{x}+\tau_{k}^{(1)} d_{k}^{(1)}\right)-f_{1}(\bar{x})<0$, for large $k$. So, there exists a subsequence of $\left\{\bar{x}+\tau_{k}^{(1)} d_{k}^{(1)}\right\}$, also denoted by $\left\{\bar{x}+\tau_{k}^{(1)} d_{k}^{(1)}\right\}$, such that $f_{1}(\bar{x}+$ 
$\left.\tau_{k}^{(1)} d_{k}^{(1)}\right)-f_{1}(\bar{x})<0$. By applying the subsequence $\left\{\bar{x}+\tau_{k}^{(1)} d_{k}^{(1)}\right\}$ for $f_{2}$ as in (3.4), we obtain a subsequence $\left\{\bar{x}+\tau_{k}^{(2)} d_{k}^{(2)}\right\}$ of $\left\{\bar{x}+\tau_{k}^{(1)} d_{k}^{(1)}\right\}$ such that

$$
\left\{\begin{array}{l}
f_{1}\left(\bar{x}+\tau_{k}^{(2)} d_{k}^{(2)}\right)-f_{1}(\bar{x})<0, \\
f_{2}\left(\bar{x}+\tau_{k}^{(2)} d_{k}^{(2)}\right)-f_{2}(\bar{x})<0 .
\end{array}\right.
$$

Continuing this process, we obtain a subsequence $\left\{\bar{x}+\tau_{k}^{(m)} d_{k}^{(m)}\right\}$ of $\left\{\bar{x}+\tau_{k} d_{k}\right\}$ in $\Omega$ such that $f_{i}\left(\bar{x}+\tau_{k}^{(m)} d_{k}^{(m)}\right)-f_{i}(\bar{x})<0$ for all $i=1, \ldots, m$, which contradicts (3.1). Hence, the claim (3.2) also holds for Case 2. Therefore, (3.2) fulfills. We conclude from (3.2) and (VC-ACQ) that

$$
\begin{aligned}
& \left(\bigcup_{i \in I} \partial^{C} f_{i}(\bar{x})\right)^{s} \cap\left(\bigcup_{t \in I_{g}} \partial^{C} g_{t}(\bar{x})\right)^{-} \cap\left(\bigcup_{i \in I_{h}} \partial^{C} h_{i}(\bar{x})\right)^{\perp} \cap\left(\bigcup_{i \in I_{0+}} \partial^{C} H_{i}(\bar{x})\right)^{\perp} \\
& \cap\left(\bigcup_{i \in I_{00} \cup I_{0-}}-\partial^{C} H_{i}(\bar{x})\right)^{-} \cap\left(\bigcup_{i \in I_{+0} \cup I_{00}} \partial^{C} G_{i}(\bar{x})\right)^{-}=\emptyset .
\end{aligned}
$$

This leads that there is no $d \in \mathbb{R}^{n}$ such that

$$
\begin{cases}\left\langle\partial^{C} f_{i}(\bar{x}), d\right\rangle<0, & \forall i \in I, \\ \left\langle\partial^{C} g_{t}(\bar{x}), d\right\rangle \leq 0, & \forall t \in I_{g}, \\ \left\langle\partial^{C} h_{i}(\bar{x}), d\right\rangle=0, & \forall i \in I_{h}, \\ \left\langle\partial^{C} H_{i}(\bar{x}), d\right\rangle=0, & \forall i \in I_{0+}, \\ \left\langle-\partial^{C} H_{i}(\bar{x}), d\right\rangle \leq 0, & \forall i \in I_{00} \cup I_{0-} \\ \left\langle\partial^{C} G_{i}(\bar{x}), d\right\rangle \leq 0, & \forall i \in I_{+0} \cup I_{00}\end{cases}
$$

On the other hand, as $\operatorname{co}\left\{\bigcup_{i \in I} \partial^{C} f_{i}(\bar{x})\right\}$ is a compact set, $\operatorname{co}\left\{\bigcup_{i \in I} \partial^{C} f_{i}(\bar{x})\right\}+\Delta_{1}$ is closed. According to Lemma 2.3, one has

$$
\begin{aligned}
0 \in & \operatorname{co\bigcup } \bigcup_{i \in I} \partial^{C} f(\bar{x})+\operatorname{pos}\left(\bigcup_{t \in I_{g}} \partial^{C} g_{t}(\bar{x}) \cup \bigcup_{i \in I_{00} \cup I_{0-}}\left(-\partial^{C} H_{i}(\bar{x})\right) \cup \bigcup_{i \in I_{+0} \cup I_{00}} \partial^{C} G_{i}(\bar{x})\right) \\
& +\operatorname{span}\left(\bigcup_{i \in I_{h}} \partial^{C} h_{i}(\bar{x}) \cup \bigcup_{i \in I_{0+}} \partial^{C} H_{i}(\bar{x})\right) .
\end{aligned}
$$

This entails that

$$
\begin{aligned}
0 \in & \operatorname{co} \bigcup_{i \in I} \partial^{C} f(\bar{x})+\operatorname{pos} \bigcup_{t \in I_{g}} \partial^{C} g_{t}(\bar{x})+\operatorname{span} \bigcup_{i \in I_{h}} \partial^{C} h_{i}(\bar{x})+\operatorname{span} \bigcup_{i \in I_{0+}} \partial^{C} H_{i}(\bar{x}) \\
& +\operatorname{pos} \bigcup_{i \in I_{00} \cup I_{0-}}\left(-\partial^{C} H_{i}(\bar{x})\right)+\operatorname{pos} \bigcup_{i \in I_{+0} \cup I_{00}} \partial^{C} G_{i}(\bar{x}) .
\end{aligned}
$$

By Lemma 2.2, we know that there exists $\left(\alpha, \lambda^{g}, \lambda^{h}, \lambda^{G}, \lambda^{H}\right) \in \mathbb{R}_{+}^{m} \times \Lambda(\bar{x}) \times \mathbb{R}^{q} \times \mathbb{R}^{l} \times \mathbb{R}^{l}$ with $\sum_{i \in I} \alpha_{i}=1, \lambda_{I_{+}}^{H}=0, \lambda_{I_{00} \cup I_{0_{-}}}^{H} \geq 0, \lambda_{I_{+-} \cup I_{0+} \cup I_{0_{-}}}^{G}=0$ and $\lambda_{I_{+0} \cup I_{00}}^{G} \geq 0$ such that

$$
0 \in \sum_{i \in I} \alpha_{i} \partial^{C} f_{i}(\bar{x})+\sum_{t \in T} \lambda_{t}^{g} \partial^{C} g_{t}(\bar{x})+\sum_{i \in I_{h}} \lambda_{i}^{h} \partial^{C} h_{i}(\bar{x})-\sum_{i \in I_{l}} \lambda_{i}^{H} \partial^{C} H_{i}(\bar{x})+\sum_{i \in I_{l}} \lambda_{i}^{G} \partial^{C} G_{i}(\bar{x}) .
$$

Hence, $\bar{x}$ is a VC-stationary point of $(\mathrm{P})$.

Proposition 3.2. Let $\bar{x}$ be a strong stationary point of $(P)$. Suppose that $\hat{I}_{0+}^{-} \cup I_{+0}^{+}=\emptyset$ and $g_{t}\left(t \in I_{g}\right), h_{i}\left(i \in I_{h}^{+}\right),-h_{i}\left(i \in I_{h}^{-}\right),-H_{i}\left(i \in \hat{I}_{0+}^{+} \cup \hat{I}_{00}^{+} \cup \hat{I}_{0-}^{+}\right)$are $\partial^{C}$-quasiconvex at $\bar{x}$ on $\Omega$. 
(i) If $f_{i}(i \in I)$ are $\partial^{C}$-pseudoconvex at $\bar{x}$ on $\Omega$, then $\bar{x}$ is an efficient solution of $(P)$.

(ii) If $f_{i}(i \in I)$ are strictly $\partial^{C}$-pseudoconvex at $\bar{x}$ on $\Omega$, then $\bar{x}$ is a weakly efficient solution of $(P)$.

Proof. Since $\bar{x}$ is a strong stationary point of (P), there exists $\left(\alpha, \lambda_{J}^{g}, \lambda^{h}, \lambda^{G}, \lambda^{H}\right) \in \mathbb{R}_{+}^{m} \times$ $\mathbb{R}_{+}^{|J|} \times \mathbb{R}^{q} \times \mathbb{R}^{l} \times \mathbb{R}^{l}$, where $J$ is a finite subset of $I_{g}$, with $\sum_{i \in I} \alpha_{i}=1, \lambda_{I_{+}}^{H}=0, \lambda_{I_{00} \cup I_{0-}}^{H} \geq 0$, $\lambda_{I_{+-} \cup I_{0+} \cup I_{00} \cup I_{0_{-}}}=0, \lambda_{I_{+0}}^{G} \geq 0$ and $\xi_{i}^{f} \in \partial^{C} f_{i}(\bar{x})(i \in I), \xi_{t}^{g} \in \partial^{C} g_{i}(\bar{x})(t \in J), \xi_{i}^{h} \in \partial^{C} h_{i}(\bar{x})(i \in$ $\left.I_{h}\right), \xi_{i}^{H} \in \partial^{C} H_{i}(\bar{x})\left(i \in I_{0+} \cup I_{00} \cup I_{0-}\right), \xi_{i}^{G} \in \partial^{C} G_{i}(\bar{x})\left(i \in I_{+0}\right)$ such that

$$
\sum_{i \in I} \alpha_{i} \xi_{i}^{f}+\sum_{t \in J} \lambda_{t}^{g} \xi_{t}^{g}+\sum_{i \in I_{h}} \lambda_{i}^{h} \xi_{i}^{h}-\sum_{i \in I_{0+} \cup I_{00} \cup I_{0-}} \lambda_{i}^{H} \xi_{i}^{H}+\sum_{i \in I_{+0}} \lambda_{i}^{G} \xi_{i}^{G}=0 .
$$

For an arbitrary $x \in \Omega$, one obtains that $g_{t}(x) \leq 0=g_{t}(\bar{x})$ for each $t \in I_{g}$. Thus, the $\partial^{C}$ quasiconvexity at $\bar{x}$ on $\Omega$ of $g_{t}\left(t \in I_{g}\right)$ give us that $\left\langle\xi_{t}^{g}, x-\bar{x}\right\rangle \leq 0, \forall t \in J$, which in turn together with $\lambda_{J}^{g} \in \mathbb{R}_{+}^{|J|}$ leads that

$$
\left\langle\sum_{t \in J} \lambda_{t}^{g} \xi_{t}^{g}, x-\bar{x}\right\rangle \leq 0
$$

We deduce from $x, \bar{x} \in \Omega$ that $h_{i}(x)=h_{i}(\bar{x})=0, \forall i \in I_{h}$. Hence,

$$
h_{i}(x) \leq h_{i}(\bar{x}), \forall i \in I_{h}^{+} \text {and }-h_{i}(x) \leq-h(\bar{x}), \forall i \in I_{h}^{-} .
$$

We deduce from the above inequalities, the $\partial^{C}$-quasiconvexity at $\bar{x}$ on $\Omega$ of $h_{i}\left(i \in I_{h}^{+}\right)$and $-h_{i}\left(i \in I_{h}^{-}\right)$and $\partial^{C}\left(-h_{i}\right)(\bar{x})=-\partial^{C} h_{i}(\bar{x})\left(i \in I_{h}^{-}\right)$that

$$
\left\langle\xi_{i}^{h}, x-\bar{x}\right\rangle \leq 0, \forall i \in I_{h}^{+} \text {and }\left\langle-\xi_{i}^{h}, x-\bar{x}\right\rangle \leq 0, \quad \forall i \in I_{h}^{-} .
$$

This, taking into account the definitions of $I_{h}^{+}, I_{h}^{-}$, results in

$$
\left\langle\sum_{i \in I_{h}} \lambda_{i}^{h} \xi_{i}^{h}, x-\bar{x}\right\rangle \leq 0
$$

Again, we derive from $x \in \Omega$ that $-H_{i}(x) \leq 0, \forall i \in I_{l}$, and thus, $-H_{i}(x) \leq-H_{i}(\bar{x}), i \in \hat{I}_{0+}^{+} \cup \hat{I}_{00}^{+} \cup$ $\hat{I}_{0-}^{+}$. Therefore, by the $\partial^{C}$-quasiconvexity of $-H_{i}, i \in \hat{I}_{0+}^{+} \cup \hat{I}_{00}^{+} \cup \hat{I}_{0-}^{+}$at $\bar{x}$ on $\Omega$, one concludes that

$$
\left\langle-\xi_{i}^{H}, x-\bar{x}\right\rangle \leq 0, \forall i \in \hat{I}_{0+}^{+} \cup \hat{I}_{00}^{+} \cup \hat{I}_{0-}^{+} .
$$

As $I_{+0}^{+} \cup \hat{I}_{0+}^{-}=\emptyset$, we infer from (3.5) - (3.8) that

$$
\begin{aligned}
\left\langle\sum_{i \in I} \alpha_{i} \xi_{i}^{f}, x-\bar{x}\right\rangle & =-\left\langle\sum_{t \in T} \lambda_{t}^{g} \xi_{t}^{g}+\sum_{i \in I_{h}} \lambda_{i}^{h} \xi_{i}^{h}-\sum_{i \in I_{0+} \cup I_{00} \cup I_{0-}} \lambda_{i}^{H} \xi_{i}^{H}+\sum_{i \in I_{+0}} \lambda_{i}^{G} \xi_{i}^{G}, x-\bar{x}\right\rangle \\
& \geq 0
\end{aligned}
$$

for all $x \in \Omega$.

(i) Suppose to the contrary that $\bar{x}$ is not a weakly efficient solution of (P). This amounts to the existence of a feasible point $\tilde{x} \in \Omega$ such that $f_{i}(\tilde{x})<f_{i}(\bar{x}), \forall i=1, \ldots, m$. The fact on $f_{i}(\tilde{x})<f_{i}(\bar{x})$ 
for each $i$ and the $\partial^{C}$-pseudoconvexity at $\bar{x}$ on $\Omega$ of $f_{i}(i \in I)$ give us the conclusion $\left\langle\xi_{i}^{f}, \tilde{x}-\bar{x}\right\rangle<$ 0 . Combining this with $\alpha \in \mathbb{R}_{+}^{m}$ and $\sum_{i=1}^{m} \alpha_{i}=1$, we arrive at

$$
\left\langle\sum_{i \in I} \alpha_{i} \xi_{i}^{f}, \tilde{x}-\bar{x}\right\rangle<0
$$

contradicting with (3.9).

(ii) Assume that $\bar{x}$ is not an efficient solution. Then there exists a feasible point $\tilde{x}$ and at least $i_{0} \in I$ satisfying

$$
\left\{\begin{array}{l}
f_{i}(\tilde{x}) \leq f_{i}(\bar{x}), \quad \forall i \in I \backslash\left\{i_{0}\right\}, \\
f_{i_{0}}(\tilde{x})<f_{i_{0}}(\bar{x}),
\end{array}\right.
$$

and hence, $\tilde{x} \neq \bar{x}$. Since $f_{i}(i \in I)$ are strictly $\partial^{C}$-pseudoconvex at $\bar{x}$ on $\Omega$ and $x \neq \bar{x}$, one has $\left\langle\xi_{i}^{f}, \tilde{x}-\bar{x}\right\rangle<0, \forall i \in I$. Employing this with $\alpha \in \mathbb{R}_{+}^{m}$ and $\sum_{i=1}^{m} \alpha_{i}=1$ brings us that

$$
\left\langle\sum_{i \in I} \alpha_{i} \xi_{i}^{f}, \tilde{x}-\bar{x}\right\rangle<0
$$

which contradicts (3.9).

Proposition 3.3. Let $\bar{x}$ be a VC-stationary point of $(P)$. Assume that $\hat{I}_{0+}^{-} \cup I_{+0}^{+} \cup I_{00}^{+}=\emptyset$ and $g_{t}\left(t \in I_{g}\right), h_{i}\left(i \in I_{h}^{+}\right),-h_{i}\left(i \in I_{h}^{-}\right),-H_{i}\left(i \in \hat{I}_{0+}^{+} \cup \hat{I}_{00}^{+} \cup \hat{I}_{0-}^{+}\right)$are $\partial^{C}$-quasiconvex at $\bar{x}$ on $\Omega$.

(i) If $f_{i}(i \in I)$ are $\partial^{C}$-pseudoconvex at $\bar{x}$, then $\bar{x}$ on $\Omega$ is an efficient solution of $(P)$.

(ii) If $f_{i}(i \in I)$ are strictly $\partial^{C}$-pseudoconvex at $\bar{x}$ on $\Omega$, then $\bar{x}$ is a weakly efficient solution of $(P)$.

Proof. The proof is analogous to those in Proposition 3.2.

Example 3.2. Let $m=n=2$ and $l=1$. Consider the following (P):

$$
\begin{array}{cc}
\mathbb{R}_{+}^{2}-\min & f(x)=\left(x_{1}^{2},\left|x_{1}\right|+x_{2}^{2}\right), \\
\text { s.t. } \quad g_{t}(x)=-t x_{1} \leq 0, t \in T=\mathbb{N}, \\
& H_{1}(x)=\left(x_{1}^{3}+2 x_{2}\right) \geq 0, \\
& G_{1}(x) H_{1}(x)=\left|x_{1}\right|\left(x_{1}^{3}+2 x_{2}\right) \leq 0 .
\end{array}
$$

Then, $\Omega=\left\{x \in \mathbb{R}^{2} \mid x_{1}>0, x_{1}^{3}+2 x_{2}=0\right\} \cup\left\{x \in \mathbb{R}^{2} \mid x_{1}=0, x_{2} \geq 0\right\}$. For $\bar{x}=(0,0) \in \Omega$, direct calculations give that

$$
\begin{aligned}
& \mathscr{T}(\Omega, \bar{x})=\left\{x \in \mathbb{R}^{2} \mid x_{1} \geq 0, x_{2}=0\right\} \cup\left\{x \in \mathbb{R}^{2} \mid x_{1}=0, x_{2} \geq 0\right\}, \\
& \partial^{C} f_{1}(\bar{x})=\{(0,0)\}, \partial^{C} f_{2}(\bar{x})=\operatorname{co}\{(1,0),(-1,0)\}=[-1,1] \times\{0\}, \\
& I_{g}=\mathbb{N}, \partial^{C} g_{t}(\bar{x})=\{(-t, 0)\}, t \in T, \\
& I_{+}=I_{0+}=I_{0-}=\emptyset, I_{00}=\{1\}, \partial^{C} G_{1}(\bar{x})=[-1,1] \times\{0\}, \partial^{C} H_{1}(\bar{x})=\{(0,2)\} \text {, } \\
& \left(\bigcup_{t \in I_{g}} \partial^{C} g_{t}(\bar{x})\right)^{-}=\left\{x \in \mathbb{R}^{2} \mid x_{1} \geq 0\right\} \\
& \left(\bigcup_{i \in I_{00}}\left(-\partial^{C} H_{i}(\bar{x})\right)^{-}=\left(-\partial^{C} H_{1}(\bar{x})\right)^{-}=\left\{x \in \mathbb{R}^{2} \mid x_{2} \geq 0\right\},\right. \\
& \left(\bigcup_{i \in I_{00}} \partial^{C} G_{i}(\bar{x})\right)^{-}=\left(\partial^{C} G_{1}(\bar{x})\right)^{-}=\left\{x \in \mathbb{R}^{2} \mid x_{1}=0\right\},
\end{aligned}
$$


and

$$
\left(\bigcup_{t \in I_{g}} \partial^{C} g_{t}(\bar{x})\right)^{-} \cap\left(\bigcup_{i \in I_{00}}\left(-\partial^{C} H_{i}(\bar{x})\right)\right)^{-} \cap\left(\bigcup_{i \in I_{00}} \partial^{C} G_{i}(\bar{x})\right)^{-}=\left\{x \in \mathbb{R}^{2} \mid x_{1}=0, x_{2} \geq 0\right\} .
$$

Hence,

$$
\left(\bigcup_{t \in I_{g}} \partial^{C} g_{t}(\bar{x})\right)^{-} \cap\left(\bigcup_{i \in I_{00}}\left(-\partial^{C} H_{i}(\bar{x})\right)\right)^{-} \cap\left(\bigcup_{i \in I_{00}} \partial^{C} G_{i}(\bar{x})\right)^{-} \subset \mathscr{T}(\Omega, \bar{x}) .
$$

Thus, (VC-ACQ) holds at $\bar{x}$. Moreover,

$$
\Delta_{1}=\operatorname{pos}\left(\bigcup_{t \in I_{g}} \partial^{C} g_{t}(\bar{x}) \cup \bigcup_{i \in I_{00}}\left(-\partial^{C} H_{i}(\bar{x})\right) \cup \bigcup_{i \in I_{00}} \partial^{C} G_{i}(\bar{x})\right)=\left\{x \in \mathbb{R}^{2} \mid x_{2} \leq 0\right\}
$$

is closed. Because of the fact $f_{i}(x) \geq f_{i}(\bar{x}), \forall i \in I, \forall x \in \Omega$, we assert that $\bar{x} \in W E(P)$. Thus, all the assumptions in Proposition 3.1 (ii) are fulfilled. Now, let $\alpha_{1}=\alpha_{2}=\frac{1}{2}, \lambda_{1}^{H}=0, \lambda_{1}^{G}=0$, and $\lambda^{g}: T \rightarrow \mathbb{R}$ be defined by

$$
\lambda^{g}(t)= \begin{cases}\frac{1}{2}, & \text { if } t=1, \\ 0, & \text { otherwise. }\end{cases}
$$

Then,

$$
\begin{aligned}
(0,0) & =\frac{1}{2}(0,0)+\frac{1}{2}(1,0)+\frac{1}{2} \cdot(-1,0)-0 \cdot(0,2)+0 \cdot(1,0) \\
& \in \frac{1}{2}(0,0)+\frac{1}{2} \cdot[-1,1] \times\{0\}+\sum_{t \in T} \lambda_{t}^{g}(-t, 0)-\lambda_{1}^{H}(0,1)+\lambda_{1}^{G} \cdot[-1,1] \times\{0\},
\end{aligned}
$$

which means that $\bar{x}$ is a VC-stationary point of (P). Furthermore, $\hat{I}_{00}^{+}=\hat{I}_{00}^{-}=I_{00}^{-}=I_{00}^{+}=\emptyset$. We can check that $g_{t}\left(t \in I_{g}\right), f_{i}(i \in I)$ are $\partial^{C}$-convex at $\bar{x}$ on $\Omega$. For instance, since

$$
f_{2}(x)-f_{2}(\bar{x})=\left|x_{1}\right|+x_{2}^{2} \geq\left\{\beta x_{1}, \beta \in[-1,1]\right\}=\left\langle\partial^{C} f_{2}(\bar{x}), x-\bar{x}\right\rangle, \forall x \in \Omega,
$$

$f_{2}$ is $\partial^{C}$-convex at $\bar{x}$ on $\Omega$. Hence, all the assumptions in Proposition 3.3 (i) are satisfied. Then, it follows that $\bar{x}$ is an efficient solution of $(\mathrm{P})$.

Example 3.3. [7] Let $n=2$ and $m=l=1$. Consider the following (P):

$$
\begin{aligned}
\mathbb{R}_{+}-\min \quad & f(x)=-x_{2}+\left|x_{2}-x_{1}\right|= \begin{cases}-x_{1}, & \text { if } x_{2} \geq x_{1}, \\
x_{1}-2 x_{2}, & \text { if } x_{2}<x_{1},\end{cases} \\
\text { s.t. } & H_{1}(x)=x_{1} \geq 0, \\
& H_{2}(x)=x_{2} \geq 0, \\
& G_{1}(x) H_{1}(x)=\left(-x_{2}\right) \cdot x_{1} \leq 0, \\
& G_{2}(x) H_{2}(x)=\left(-x_{1}\right) \cdot x_{2} \leq 0 .
\end{aligned}
$$

Then, $\Omega=\left\{x \in \mathbb{R}^{2} \mid x_{1} \geq 0, x_{2}=0\right\} \cup\left\{x \in \mathbb{R}^{2} \mid x_{1}=0, x_{2} \geq 0\right\}$. For $\bar{x}=(0,0) \in \Omega$, we have $\mathscr{T}(\Omega, \bar{x})=\Omega$,

$$
\begin{gathered}
\partial^{C} f(\bar{x})=\operatorname{co}\{(-1,0),(1,-2)\}=\{(-1,0)+\beta((1,-2)-(-1,0)), \\
\beta \in[0,1]\}=\{(-1+2 \beta,-2 \beta), \beta \in[0,1]\}, \\
I_{+}=I_{0+}=I_{0-}=\emptyset, I_{00}=\{1,2\}, \partial^{C} G_{1}(\bar{x})=\{(0,-1)\}, \partial^{C} H_{1}(\bar{x})=\{(1,0)\}, \\
\partial^{C} G_{2}(\bar{x})=\{(-1,0)\}, \partial^{C} H_{2}(\bar{x})=\{(0,1)\}
\end{gathered}
$$


and

$$
\left(\bigcup_{i \in I_{00}}-\partial^{C} H_{i}(\bar{x})\right)^{-}=\left(\left\{((-1,0,(0,-1)\})^{-}=\mathbb{R}_{+}^{2},\left(\bigcup_{i \in I_{00}} \partial^{C} G_{i}(\bar{x})\right)^{-}=\left(\left\{((-1,0,(0,-1)\})^{-}=\mathbb{R}_{+}^{2} .\right.\right.\right.\right.
$$

Hence,

$$
\left(\bigcup_{i \in I_{00}}\left(-\partial^{C} H_{i}(\bar{x})\right)\right)^{-} \not \subset \mathscr{T}(\Omega, \bar{x}),\left(\bigcup_{i \in I_{00}}\left(-\partial^{C} H_{i}(\bar{x})\right)\right)^{-} \cap\left(\bigcup_{i \in I_{00}} \partial^{C} G_{i}(\bar{x})\right)^{-} \not \subset \mathscr{T}(\Omega, \bar{x}),
$$

i.e., both (ACQ) and (VC-ACQ) do not hold at $\bar{x}$.

We can apply Proposition 3.1 to verify that both (ACQ) and (VC-ACQ) do not hold at $\bar{x}$ as follows. Since

$$
(0,0)=(-1+2 \beta,-2 \beta)-\lambda_{1}^{H}(1,0)-\lambda_{2}^{H}(0,1)+\lambda_{1}^{G}(0,-1)+\lambda_{2}^{G}(-1,0), \beta \in[0,1],
$$

where $\lambda_{1}^{H}, \lambda_{2}^{H}, \lambda_{1}^{G}, \lambda_{2}^{G} \in \mathbb{R}_{+}$, we have

$$
\lambda_{1}^{H}+\lambda_{2}^{H}+\lambda_{1}^{G}+\lambda_{2}^{G}=-1, \lambda_{1}^{H}, \lambda_{2}^{H}, \lambda_{1}^{G}, \lambda_{2}^{G} \in \mathbb{R}_{+} .
$$

which has no solution. Hence $\bar{x}$ is not a strong stationary point of $(\mathrm{P})$, neither is $\bar{x}$ a VCstationary point of $(\mathrm{P})$. Moreover, since $\Delta=\Delta_{1}=-\mathbb{R}_{+}^{2}$ are closed and $\bar{x}=(0,0)$ is a solution of (P), we deduce from Proposition 3.1 that both (ACQ) and (VC-ACQ) do not hold at $\bar{x}$.

In addition, it should be noted that the smoothness condition of $f($.$) cannot be replaced by its$ Lipschitzian condition in [7, Theorem 4.1]. However, the smoothness condition of $f($.$) could$ be replaced by its Lipschitzian condition in Proposition 3.1 due to the assumptions (ACQ) and (VC-ACQ), which are stronger than (WGCQ) in [7], utilized in Proposition 3.1.

\section{DuAlity}

In this section, we consider the Wolfe [34] and Mond-Weir [35] duality schemes for (P). For $\bar{x} \in \Omega$, the index sets with respect to $\bar{x}$ are denoted identically to Section 3 . In what follows, for $u, v \in \mathbb{R}^{m}$, we use the notations:

$$
\begin{gathered}
u \prec v \Leftrightarrow u_{i}<v_{i}, \text { for all } i \in I, u \nprec v \text { is the negation of } u \prec v, \\
u \preceq v \Leftrightarrow\left\{\begin{array}{l}
u_{i} \leq v_{i}, \quad \text { for all } i \in I, \\
u_{i}<v_{i}, \quad \text { for at least one } i_{0} \in I, \quad u \npreceq v \text { is the negation of } u \preceq v .
\end{array}\right.
\end{gathered}
$$

Note that $\bar{x} \in \operatorname{loc} E(P)(\bar{x} \in \operatorname{loc} W E(P))$ if there exists $U \in \mathscr{U}(\bar{x})$ such that there is no $x \in \Omega \cap U$ satisfying $f(x) \preceq f(\bar{x})(f(x) \prec f(\bar{x}))$.

4.1. The Mond-Weir type duality. For $\bar{x} \in \Omega,\left(u, \alpha, \lambda^{g}, \lambda^{h}, \lambda^{G}, \lambda^{H}\right) \in \mathbb{R}^{n} \times \mathbb{R}_{+}^{m} \times \mathbb{R}_{+}^{|T|} \times \mathbb{R}^{q} \times$ $\mathbb{R}^{l} \times \mathbb{R}^{l}$ with $\sum_{i \in I} \alpha_{i}=1, \lambda_{I_{+}(\bar{x})}^{H} \geq 0, \lambda_{I_{0+}(\bar{x})}^{G} \leq 0$ and $\lambda_{I_{+-}(\bar{x}) \cup I_{0-}(\bar{x})} \geq 0$, let us define

$$
\widetilde{L}\left(u, \alpha, \lambda^{g}, \lambda^{h}, \lambda^{G}, \lambda^{H}\right):=f(u) .
$$

In the line of $[9,23]$, we consider the Mond-Weir type dual problem as follows:

$\left(\mathrm{D}_{M W}(\bar{x})\right): \mathbb{R}_{+}^{m}-\max \widetilde{L}\left(u, \alpha, \lambda^{g}, \lambda^{h}, \lambda^{G}, \lambda^{H}\right)=f(u)$

$$
\begin{aligned}
& \text { s.t. } 0 \in \sum_{i \in I} \alpha_{i} \partial^{C} f_{i}(u)+\sum_{t \in T} \lambda_{t}^{g} \partial^{C} g_{t}(u)+\sum_{i \in I_{h}} \lambda_{i}^{h} \partial^{C} h_{i}(u)-\sum_{i \in I_{l}} \lambda_{i}^{H} \partial^{C} H_{i}(u)+\sum_{i \in I_{l}} \lambda_{i}^{G} \partial^{C} G_{i}(u), \\
& \begin{array}{c}
\lambda_{t}^{g} g_{t}(u) \geq 0(t \in T), \lambda_{i}^{h} h_{i}(u)=0\left(i \in I_{h}\right),-\lambda_{i}^{H} H_{i}(u) \geq 0\left(i \in I_{l}\right), \lambda_{i}^{G} G_{i}(u) \geq 0\left(i \in I_{l}\right), \\
\sum_{i \in I} \alpha_{i}=1, \lambda_{I_{+}(\bar{x})}^{H} \geq 0, \lambda_{I_{0+}(\bar{x})}^{G} \leq 0, \lambda_{I_{+-}(\bar{x}) \cup I_{0-}(\bar{x})} \geq 0, \\
\left(u, \alpha, \lambda^{g}, \lambda^{h}, \lambda^{G}, \lambda^{H}\right) \in \mathbb{R}^{n} \times \mathbb{R}_{+}^{m} \times \mathbb{R}_{+}^{|T|} \times \mathbb{R}^{q} \times \mathbb{R}^{l} \times \mathbb{R}^{l} .
\end{array}
\end{aligned}
$$


The feasible set of $\left(\mathrm{D}_{M W}(\bar{x})\right)$ is defined by

$$
\begin{gathered}
\Omega_{M W}(\bar{x}):=\left\{\left(u, \alpha, \lambda^{g}, \lambda^{h}, \lambda^{G}, \lambda^{H}\right) \in \mathbb{R}^{n} \times \mathbb{R}_{+}^{m} \times \mathbb{R}_{+}^{|T|} \times \mathbb{R}^{q} \times \mathbb{R}^{l} \times \mathbb{R}^{l} \mid\right. \\
0 \in \sum_{i \in I} \alpha_{i} \partial^{C} f_{i}(u)+\sum_{t \in T} \lambda_{t}^{g} \partial^{C} g_{t}(u)+\sum_{i \in I_{h}} \lambda_{i}^{h} \partial^{C} h_{i}(u)-\sum_{i \in I_{l}} \lambda_{i}^{H} \partial^{C} H_{i}(u)+\sum_{i \in I_{l}} \lambda_{i}^{G} \partial^{C} G_{i}(u), \\
\lambda_{t}^{g} g_{t}(u) \geq 0(t \in T), \lambda_{i}^{h} h_{i}(u)=0\left(i \in I_{h}\right),-\lambda_{i}^{H} H_{i}(u) \geq 0\left(i \in I_{l}\right), \lambda_{i}^{G} G_{i}(u) \geq 0\left(i \in I_{l}\right) \\
\left.\sum_{i \in I} \alpha_{i}=1, \lambda_{I_{+}(\bar{x})}^{H} \geq 0, \lambda_{I_{0+}(\bar{x})}^{G} \leq 0, \lambda_{I_{+-}(\bar{x}) \cup I_{0-}(\bar{x})}^{G} \geq 0,\right\} .
\end{gathered}
$$

We designate by

$$
\operatorname{pr}_{\mathbb{R}^{n}} \Omega_{M W}(\bar{x}):=\left\{u \in \mathbb{R}^{n} \mid\left(u, \alpha, \lambda^{g}, \lambda^{h}, \lambda^{G}, \lambda^{H}\right) \in \Omega_{M W}(\bar{x})\right\}
$$

the projection of the set $\Omega(\bar{x})$ on $\mathbb{R}^{n}$. The other Mond-Weir type duality problem of $(\mathrm{P})$, which is not dependent on $\bar{x}$, is

$\left(\mathrm{D}_{M W}\right): \mathbb{R}_{+}^{m}-\max \widetilde{L}\left(y, \alpha, \lambda^{g}, \lambda^{h}, \lambda^{G}, \lambda^{H}\right)=f(y)$

s.t. $\left(y, \alpha, \lambda^{g}, \lambda^{h}, \lambda^{G}, \lambda^{H}\right) \in \Omega_{M W}:=\bigcap_{\bar{x} \in \Omega} \Omega_{M W}(\bar{x})$.

Definition 4.1. Let $\bar{x} \in \Omega$.

(i) $\left(\bar{u}, \bar{\alpha}, \bar{\lambda}^{g}, \bar{\lambda}^{h}, \bar{\lambda}^{G}, \bar{\lambda}^{H}\right) \in \Omega_{M W}(\bar{x})$ is said to be a locally efficient solution of $\left(\mathrm{D}_{M W}(\bar{x})\right)$, denoted by $\left(\bar{u}, \bar{\alpha}, \bar{\lambda}^{g}, \bar{\lambda}^{h}, \bar{\lambda}^{G}, \bar{\lambda}^{H}\right) \in \operatorname{loc} E\left(\mathrm{D}_{M W}(\bar{x})\right)$, if there exists $U \in \mathscr{N}(\bar{u})$ such that there is no point $\left(u, \alpha, \lambda^{g}, \lambda^{h}, \lambda^{G}, \lambda^{H}\right) \in \Omega_{M W}(\bar{x}) \cap U$ satisfying

$$
\widetilde{L}\left(\bar{u}, \bar{\alpha}, \bar{\lambda}^{g}, \bar{\lambda}^{h}, \bar{\lambda}^{G}, \bar{\lambda}^{H}\right) \preceq \widetilde{L}\left(u, \alpha, \lambda^{g}, \lambda^{h}, \lambda^{G}, \lambda^{H}\right) .
$$

(ii) $\left(\bar{u}, \bar{\alpha}, \bar{\lambda}^{g}, \bar{\lambda}^{h}, \bar{\lambda}^{G}, \bar{\lambda}^{H}\right) \in \Omega_{M W}(\bar{x})$ is called a locally weakly efficient solution of $\left(\mathrm{D}_{M W}(\bar{x})\right)$, denoted by $\left(\bar{u}, \bar{\alpha}, \bar{\lambda}^{g}, \bar{\lambda}^{h}, \bar{\lambda}^{G}, \bar{\lambda}^{H}\right) \in \operatorname{loc} W E\left(\mathrm{D}_{M W}(\bar{x})\right)$, if there exists $U \in \mathscr{N}(\bar{u})$ such that there is no $\left(u, \alpha, \lambda^{g}, \lambda^{h}, \lambda^{G}, \lambda^{H}\right) \in \Omega_{M W}(\bar{x}) \cap U$ fulfilling

$$
\widetilde{L}\left(\bar{u}, \bar{\alpha}, \bar{\lambda}^{g}, \bar{\lambda}^{h}, \bar{\lambda}^{G}, \bar{\lambda}^{H}\right) \prec \widetilde{L}\left(u, \alpha, \lambda^{g}, \lambda^{h}, \lambda^{G}, \lambda^{H} .\right.
$$

(iii) $\left(\bar{y}, \bar{\alpha}, \bar{\lambda}^{g}, \bar{\lambda}^{h}, \bar{\lambda}^{G}, \bar{\lambda}^{H}\right) \in \Omega_{M W}$ is a locally efficient solution of $\left(\mathrm{D}_{M W}\right)$, denoted by $\left(\bar{y}, \bar{\alpha}, \bar{\lambda}^{g}, \bar{\lambda}^{h}\right.$, $\left.\bar{\lambda}^{G}, \bar{\lambda}^{H}\right) \in \operatorname{loc} E\left(\mathrm{D}_{M W}\right)$, if there exists $U \in \mathscr{N}(\bar{y})$ such that there is no $\left(y, \alpha, \lambda^{g}, \lambda^{h}, \lambda^{G}, \lambda^{H}\right) \in$ $\Omega_{M W} \cap U$ fulfilling

$$
\widetilde{L}\left(\bar{y}, \bar{\alpha}, \bar{\lambda}^{g}, \bar{\lambda}^{h}, \bar{\lambda}^{G}, \bar{\lambda}^{H}\right) \preceq \widetilde{L}\left(y, \alpha, \lambda^{g}, \lambda^{h}, \lambda^{G}, \lambda^{H}\right) .
$$

(iv) $\left(\bar{y}, \bar{\alpha}, \bar{\lambda}^{g}, \bar{\lambda}^{h}, \bar{\lambda}^{G}, \bar{\lambda}^{H}\right) \in \Omega_{M W}$ is a locally weakly efficient solution of (D $\left.\mathrm{D}_{M W}\right)$, denoted by $\left(\bar{y}, \bar{\alpha}, \bar{\lambda}^{g}, \bar{\lambda}^{h}, \bar{\lambda}^{G}, \bar{\lambda}^{H}\right) \in \operatorname{loc} W E\left(\mathrm{D}_{M W}\right)$, if there exists $U \in \mathscr{N}(\bar{y})$ such that there is no $\left(y, \alpha, \lambda^{g}, \lambda^{h}, \lambda^{G}, \lambda^{H}\right) \in \Omega_{M W} \cap U$ satisfying

$$
\widetilde{L}\left(\bar{y}, \bar{\alpha}, \bar{\lambda}^{g}, \bar{\lambda}^{h}, \bar{\lambda}^{G}, \bar{\lambda}^{H}\right) \prec \widetilde{L}\left(y, \alpha, \lambda^{g}, \lambda^{h}, \lambda^{G}, \lambda^{H}\right) .
$$

If $U=\mathbb{R}^{n}$, the word "locally" is dropped.

Proposition 4.1. (Weak Duality) Let $x \in \Omega$ and $\left(y, \alpha, \lambda^{g}, \lambda^{h}, \lambda^{G}, \lambda^{H}\right) \in \Omega_{M W}$. Suppose that $g_{t}\left(t \in I_{g}^{+}(x)\right), h_{i}\left(i \in I_{h}^{+}(x)\right),-h_{i}\left(i \in I_{h}^{-}(x)\right), H_{i}\left(i \in \hat{I}_{0}^{-}(x)\right),-H_{i}\left(i \in \hat{I}_{+}^{+}(x) \cup \hat{I}_{0}^{+}(x)\right), G_{i}\left(i \in I_{+0}^{+}(x) \cup\right.$ $\left.I_{+-}^{+}(x) \cup I_{00}^{+}(x) \cup I_{0-}^{+}(x)\right),-G_{i}\left(i \in I_{+0}^{-}(x) \cup I_{0+}^{-}(x) \cup I_{00}^{-}(x)\right)$ are $\partial^{C}$-quasiconvex at $y$ on $X_{M W}:=$ $\Omega \cup \operatorname{pr}_{\mathbb{R}^{n}} \Omega_{M W}$.

(i) If $f_{i}(i \in I)$ are $\partial^{C}$-pseudoconvex at $y$ on $X_{M W}$, then $f(x) \nprec \widetilde{L}\left(y, \lambda^{g}, \lambda^{h}, \lambda^{G}, \lambda^{H}\right)$.

(ii) If $f_{i}(i \in I)$ are strictly $\partial^{C}$-pseudoconvex at $y$ on $X_{M W}$, then $f(x) \npreceq \widetilde{L}\left(y, \lambda^{g}, \lambda^{h}, \lambda^{G}, \lambda^{H}\right)$. 
Proof. For $x \in \Omega$ and $\left(y, \alpha, \lambda^{g}, \lambda^{h}, \lambda^{G}, \lambda^{H}\right) \in \Omega_{M W}=\bigcap_{\bar{x} \in \Omega} \Omega_{M W}(\bar{x})$, we have

$$
\begin{gathered}
g_{t}(x) \leq 0(t \in T), h_{i}(x)=0\left(i \in I_{h}\right), H_{i}(x) \geq 0\left(i \in I_{l}\right), G_{i}(x) H_{i}(x) \leq 0\left(i \in I_{l}\right), \\
0 \in \sum_{i \in I} \alpha_{i} \partial^{C} f_{i}(y)+\sum_{t \in T} \lambda_{t}^{g} \partial^{C} g_{t}(y)+\sum_{i \in I_{h}} \lambda_{i}^{h} \partial^{C} h_{i}(y)-\sum_{i \in I_{l}} \lambda_{i}^{H} \partial^{C} H_{i}(y)+\sum_{i \in I_{l}} \lambda_{i}^{G} \partial^{C} G_{i}(y),
\end{gathered}
$$

and

$$
\lambda_{t}^{g} g_{t}(y) \geq 0(t \in T), \lambda_{i}^{h} h_{i}(y)=0\left(i \in I_{h}\right),-\lambda_{i}^{H} H_{i}(y) \geq 0\left(i \in I_{l}\right), \lambda_{i}^{G} G_{i}(y) \geq 0\left(i \in I_{l}\right),
$$

with $\sum_{i \in I} \alpha_{i}=1, \lambda_{I_{+}(x)}^{H} \geq 0, \lambda_{I_{0+}(x)}^{G} \leq 0, \lambda_{I_{+-}(x) \cup I_{0-}(x)}^{G} \geq 0$. We deduce from (4.2) that there exist $\xi_{i}^{f} \in \partial^{C} f_{i}(y)(i \in I), \xi_{t}^{g} \in \partial^{C} g_{t}(y)(t \in T), \xi_{i}^{h} \in \partial^{C} h_{i}(y)\left(i \in I_{h}\right), \xi_{i}^{H} \in \partial^{C} H_{i}(y)\left(i \in I_{l}\right), \xi_{i}^{G} \in$ $\partial^{C} G_{i}(y)\left(i \in I_{l}\right)$ such that

$$
\sum_{i \in I} \alpha_{i} \xi_{i}^{f}+\sum_{t \in J} \lambda_{t}^{g} \xi_{t}^{g}+\sum_{i \in I_{h}} \lambda_{i}^{h} \xi_{i}^{h}-\sum_{i \in I_{y}} \lambda_{i}^{H} \xi_{i}^{H}+\sum_{i \in I_{y}} \lambda_{i}^{G} \xi_{i}^{G}=0 .
$$

It follows from (4.1) and (4.3) that

$$
\begin{gathered}
g_{t}(x) \leq 0 \leq g_{t}(y), \forall t \in I_{g}^{+}(x), \\
h_{i}(x)=h_{i}(y)=0, \forall i \in I_{h}^{+}(x) \cup I_{h}^{-}(x), \\
H_{i}(x)=0 \leq H_{i}(y), \forall i \in \hat{I}_{0}^{-}(x), \\
-H_{i}(x) \leq 0 \leq-H_{i}(y), \forall i \in \hat{I}_{+}^{+}(x) \cup \hat{I}_{0}^{+}(x), \\
G_{i}(x) \leq 0 \leq G_{i}(y), \forall i \in I_{+0}^{+}(x) \cup I_{+-}^{+}(x) \cup I_{00}^{+}(x) \cup I_{0-}^{+}(x),
\end{gathered}
$$

and

$$
-G_{i}(x) \leq 0 \leq-G_{i}(y), \forall i \in I_{+0}^{-}(x) \cup I_{0+}^{-}(x) \cup I_{00}^{-}(x) .
$$

Thus, we deduce from the above inequalities, the $\partial^{C}$-quasiconvexity of $g_{t}\left(t \in I_{g}^{+}(x)\right), h_{i}(i \in$ $\left.I_{h}^{+}(x)\right),-h_{i}\left(i \in I_{h}^{-}(x)\right), H_{i}\left(i \in \hat{I}_{0}^{-}(x)\right),-H_{i}\left(i \in \hat{I}_{+}^{+}(x) \cup \hat{I}_{0}^{+}(x)\right), G_{i}\left(i \in I_{+0}^{+}(x) \cup I_{+-}^{+}(x) \cup I_{00}^{+}(x) \cup\right.$ $\left.I_{0-}^{+}(x)\right),-G_{i}\left(i \in I_{+0}^{-}(x) \cup I_{0+}^{-}(x) \cup I_{00}^{-}(x)\right)$ at $y$ on $X_{M W}$, and the definitions of the index sets that

$$
\begin{gathered}
\left\langle\xi_{t}^{g}, x-y\right\rangle \leq 0, \lambda_{t}^{g}>0, \forall t \in I_{g}^{+}(x), \\
\left\langle\xi_{i}^{h}, x-y\right\rangle \leq 0, \lambda_{i}^{h}>0, \forall i \in I_{h}^{+}(x), \\
\left\langle-\xi_{i}^{h}, x-y\right\rangle \leq 0, \lambda_{i}^{h}<0, \forall i \in I_{h}^{-}(x), \\
\left\langle\xi_{i}^{H}, x-y\right\rangle \leq 0, \lambda_{i}^{H}<0, \forall i \in \hat{I}_{0}^{-}(x), \\
\left\langle-\xi_{i}^{H}, x-y\right\rangle \leq 0, \lambda_{i}^{H}>0, \forall i \in \hat{I}_{+}^{+}(x) \cup \hat{I}_{0}^{+}(x), \\
\left\langle\xi_{i}{ }^{G}, x-y\right\rangle \leq 0, \lambda_{i}^{G}>0, \forall i \in I_{+0}^{+}(x) \cup I_{+-}^{+}(x) \cup I_{00}^{+}(x) \cup I_{0-}^{+}(x),
\end{gathered}
$$

and

$$
\left\langle-\xi_{i}^{G}, x-y\right\rangle \leq 0, \lambda_{i}^{G}<0, \forall i \in I_{+0}^{-}(x) \cup I_{0+}^{-}(x) \cup I_{00}^{-}(x) .
$$

Employing this together with (4.4) gives us the inequality

$$
\left\langle\sum_{i \in I} \alpha_{i} \xi_{i}^{f}, x-y\right\rangle=-\left\langle\sum_{t \in T} \lambda_{t}^{g} \xi_{t}^{g}+\sum_{i \in I_{h}} \lambda_{i}^{h} \xi_{i}^{h}-\sum_{i \in I_{l}} \lambda_{i}^{H} \xi_{i}^{H}+\sum_{i \in I_{l}} \lambda_{i}^{G} \xi_{i}^{G}, x-y\right\rangle \geq 0 .
$$

(i) Suppose by contradiction that $f(x) \prec L\left(y, \alpha, \lambda^{g}, \lambda^{h}, \lambda^{G}, \lambda^{H}\right)$, equivalently, $f_{i}(x)<f_{i}(y)$, $i \in I$. The above inequalities and the pseudoconvexity of $f_{i}(i \in I)$ at $y$ on $X_{M W}$ tell us that 
$\left\langle\xi_{i}^{f}, x-y\right\rangle<0, \forall i \in I$, which along with $\alpha \in \mathbb{R}_{+}^{m}$ and $\sum_{i \in I} \alpha_{i}=1$ leads that $\left\langle\sum_{i=1}^{m} \alpha_{i} \xi_{i}^{f}, x-y\right\rangle<0$, contradicting (4.5).

(ii) Assume by contradiction that $f(x) \preceq L\left(y, \alpha, \lambda^{g}, \lambda^{h}, \lambda^{G}, \lambda^{H}\right)$. This is equivalent to

$$
\begin{cases}f_{i}(x) \leq f_{i}(y), & \forall i \in I, \\ f_{i_{0}}(x)<f_{i_{0}}(y), & \text { for at least one } i_{0} \in I,\end{cases}
$$

which implies $x \neq y$. Granting this, we can deduce from the strictly pseudoconvexity of $f_{i}(i \in I)$ at $y$ on $X_{M W}$ that $\left\langle\xi_{i}^{f}, x-y\right\rangle<0, \forall i \in I$. This, taking into account $\alpha \in \mathbb{R}_{+}^{m}$ and $\sum_{i \in I} \alpha_{i}=1$, yields that $\left\langle\sum_{i=1}^{m} \alpha_{i} \xi_{i}^{f}, x-y\right\rangle<0$, contradicting with (4.5).

Corollary 4.1. (Weak Duality) Let $\bar{x} \in \Omega$ and $\left(u, \alpha, \lambda^{g}, \lambda^{h}, \lambda^{G}, \lambda^{H}\right) \in \Omega_{M W}(\bar{x})$. Suppose that $g_{t}\left(t \in I_{g}^{+}(\bar{x})\right), h_{i}\left(i \in I_{h}^{+}(\bar{x})\right),-h_{i}\left(i \in I_{h}^{-}(\bar{x})\right), H_{i}\left(i \in \hat{I}_{0}^{-}(\bar{x})\right),-H_{i}\left(i \in \hat{I}_{+}^{+}(\bar{x}) \cup \hat{I}_{0}^{+}(\bar{x})\right), G_{i}\left(i \in I_{+0}^{+}(\bar{x}) \cup\right.$ $\left.I_{+-}^{+}(\bar{x}) \cup I_{00}^{+}(\bar{x}) \cup I_{0-}^{+}(\bar{x})\right),-G_{i}\left(i \in I_{+0}^{-}(\bar{x}) \cup I_{0+}^{-}(\bar{x}) \cup I_{00}^{-}(\bar{x})\right)$ are quasiconvex at $u$ on $\bar{X}_{M W}:=$ $\Omega \cup \Omega_{M W}(\bar{x})$.

(i) If $f_{i}(i \in I)$ are pseudoconvex at $u$ on $\bar{X}_{M W}$, then $f(\bar{x}) \nprec \widetilde{L}\left(u, \lambda^{g}, \lambda^{h}, \lambda^{G}, \lambda^{H}\right)$.

(ii) If $f_{i}(i \in I)$ are strictly pseudoconvex at $u$ on $\bar{X}_{M W}$, then $f(\bar{x}) \npreceq \widetilde{L}\left(u, \lambda^{g}, \lambda^{h}, \lambda^{G}, \lambda^{H}\right)$.

Proposition 4.2. (Strong Duality) Let $\bar{x} \in \Omega$ be a locally weakly efficient solution of $(P)$. If (VC-ACQ) holds at $\bar{x}$ and the set $\Delta_{1}$ is closed, then there exists $\left(\bar{\alpha}, \bar{\lambda}^{g}, \bar{\lambda}^{h}, \bar{\lambda}^{G}, \bar{\lambda}^{H}\right) \in \mathbb{R}_{+}^{m} \times$ $\mathbb{R}_{+}^{|T|} \times \mathbb{R}^{q} \times \mathbb{R}^{l} \times \mathbb{R}^{l}$ with $\sum_{i \in I} \bar{\alpha}_{i}=1, \bar{\lambda}_{I_{+}(\bar{x})}^{H}=0, \bar{\lambda}_{I_{00}(\bar{x}) \cup I_{0_{-}(\bar{x})}}^{H} \geq 0, \bar{\lambda}_{I_{+-}(\bar{x}) \cup I_{0+}(\bar{x}) \cup I_{0-}(\bar{x})}^{G}=0$ and $\bar{\lambda}_{I_{+0}(\bar{x}) \cup I_{00}(\bar{x})}^{G} \geq 0$ such that $\left(\bar{x}, \bar{\alpha}, \bar{\lambda}^{g}, \bar{\lambda}^{h}, \bar{\lambda}^{G}, \bar{\lambda}^{H}\right) \in \Omega_{M W}(\bar{x})$. Assume further that $g_{t}\left(t \in I_{g}^{+}(\bar{x})\right)$, $h_{i}\left(i \in I_{h}^{+}(\bar{x})\right),-h_{i}\left(i \in I_{h}^{-}(\bar{x})\right), H_{i}\left(i \in \hat{I}_{0}^{-}(\bar{x})\right),-H_{i}\left(i \in \hat{I}_{+}^{+}(\bar{x}) \cup \hat{I}_{0}^{+}(\bar{x})\right), G_{i}\left(i \in I_{+0}^{+}(\bar{x}) \cup I_{+-}^{+}(\bar{x}) \cup\right.$ $\left.I_{00}^{+}(\bar{x}) \cup I_{0-}^{+}(\bar{x})\right),-G_{i}\left(i \in I_{+0}^{-}(\bar{x}) \cup I_{0+}^{-}(\bar{x}) \cup I_{00}^{-}(\bar{x})\right)$ are $\partial^{C}$-quasiconvex at $\bar{x}$ on $\bar{X}_{M W}$.

(i) If $f_{i}(i \in I)$ are $\partial^{C}$-pseudoconvex at $\bar{x}$ on $\bar{X}_{M W}$, then $\left(\bar{x}, \bar{\alpha}, \bar{\lambda}^{g}, \bar{\lambda}^{h}, \bar{\lambda}^{G}, \bar{\lambda}^{H}\right)$ is a weakly efficient solution of $D_{M W}(\bar{x})$.

(ii) If $f_{i}(i \in I)$ are strictly $\partial^{C}$-pseudoconvex at $\bar{x}$ on $\bar{X}_{M W}$, then $\left(\bar{x}, \bar{\alpha}, \bar{\lambda}^{g}, \bar{\lambda}^{h}, \bar{\lambda}^{G}, \bar{\lambda}^{H}\right)$ is an efficient solution of $D_{M W}(\bar{x})$.

Proof. By invoking Proposition 3.1 (ii), there exists $\left(\bar{\alpha}, \bar{\lambda}^{g}, \bar{\lambda}^{h}, \bar{\lambda}^{G}, \bar{\lambda}^{H}\right) \in \mathbb{R}_{+}^{m} \times \Lambda(\bar{x}) \times \mathbb{R}^{q} \times$ $\mathbb{R}^{l} \times \mathbb{R}^{l}$ with $\sum_{i \in I} \bar{\alpha}_{i}=1, \bar{\lambda}_{I_{+}(\bar{x})}^{H}=0, \bar{\lambda}_{I_{00}(\bar{x}) \cup I_{0-}(\bar{x})}^{H} \geq 0, \bar{\lambda}_{I_{+-}(\bar{x}) \cup I_{0+}(\bar{x}) \cup I_{0-}(\bar{x})}^{G}=0$ and $\bar{\lambda}_{I_{+0}(\bar{x}) \cup I_{00}(\bar{x})} \geq 0$ such that

$$
0 \in \sum_{i \in I} \bar{\alpha}_{i} \partial^{C} f_{i}(\bar{x})+\sum_{t \in T} \bar{\lambda}_{t}^{g} \partial^{C} g_{t}(\bar{x})+\sum_{i \in I_{h}} \bar{\lambda}_{i}^{h} \partial^{C} h_{i}(\bar{x})-\sum_{i \in I_{l}} \bar{\lambda}_{i}^{H} \partial^{C} H_{i}(\bar{x})+\sum_{i \in I_{l}} \bar{\lambda}_{i}^{G} \partial^{C} G_{i}(\bar{x}) .
$$

Since $\bar{\lambda}^{g} \in \Lambda(\bar{x})$, one has $\bar{\lambda}_{t}^{g} g_{t}(\bar{x})=0$ for all $t \in T$. The fact that $\bar{x} \in \Omega$ guarantees that $\lambda_{i}^{h} h_{i}(\bar{x})=0, \forall i \in I_{h}$. Furthermore, we deduce from $\bar{\lambda}_{I_{+}(\bar{x})}^{H}=0$ and $H_{i}(\bar{x})=0$ for all $i \in I_{0}(\bar{x})$ that $-\bar{\lambda}_{i}^{H} H_{i}(\bar{x})=0$ for all $i \in I_{l}$. In addition, we obtain from $\bar{\lambda}_{I_{+-}(\bar{x}) \cup I_{0+}(\bar{x}) \cup I_{0-}(\bar{x})}=0$ and $G_{i}(\bar{x})=0$ for all $i \in I_{+0}(\bar{x}) \cup I_{00}(\bar{x})$ that $\bar{\lambda}_{i}^{G} G_{i}(\bar{x})=0$ for all $i \in I_{l}$. Thus, $\left(\bar{x}, \bar{\alpha}, \bar{\lambda}^{g}, \bar{\lambda}^{h}, \bar{\lambda}^{G}, \bar{\lambda}^{H}\right) \in \Omega_{M W}(\bar{x})$ and $f(\bar{x})=\widetilde{L}\left(\bar{x}, \bar{\alpha}, \bar{\lambda}^{g}, \bar{\lambda}^{h}, \bar{\lambda}^{G}, \bar{\lambda}^{H}\right)$.

(i) Arguing by contradiction, suppose that $\left(\bar{x}, \bar{\alpha}, \bar{\lambda}^{g}, \bar{\lambda}^{h}, \bar{\lambda}^{G}, \bar{\lambda}^{H}\right)$ is not a weakly efficient solution of $D_{M W}(\bar{x})$. By denotation, there exists $\left(u, \alpha, \lambda^{g}, \lambda^{h}, \lambda^{G}, \lambda^{H}\right) \in \Omega_{M W}(\bar{x})$ such that

$$
f(\bar{x})=\widetilde{L}\left(\bar{x}, \bar{\lambda}^{g}, \bar{\lambda}^{h}, \bar{\lambda}^{G}, \bar{\lambda}^{H}\right) \prec \widetilde{L}\left(u, \lambda^{g}, \lambda^{h}, \lambda^{G}, \lambda^{H}\right),
$$


which contradicts Corollary 4.1 (i), and thus, completes the proof.

(ii) Suppose to the contrary that $\left(\bar{x}, \bar{\alpha}, \bar{\lambda}^{g}, \bar{\lambda}^{h}, \bar{\lambda}^{G}, \bar{\lambda}^{H}\right)$ is not an efficient solution of $D_{M W}(\bar{x})$. In other words, there exists $\left(u, \alpha, \lambda^{g}, \lambda^{h}, \lambda^{G}, \lambda^{H}\right) \in \Omega_{M W}(\bar{x})$ such that

$$
f(\bar{x})=\widetilde{L}\left(\bar{x}, \bar{\lambda}^{g}, \bar{\lambda}^{h}, \bar{\lambda}^{G}, \bar{\lambda}^{H}\right) \preceq \widetilde{L}\left(u, \lambda^{g}, \lambda^{h}, \lambda^{G}, \lambda^{H}\right),
$$

which contradicts with Corollary 4.1 (ii). So, we arrive at the conclusion.

4.2. The Wolfe type duality. For $\bar{x} \in \Omega,\left(u, \alpha, \lambda^{g}, \lambda^{h}, \lambda^{G}, \lambda^{H}\right) \in \mathbb{R}^{n} \times \mathbb{R}_{+}^{m} \times \mathbb{R}_{+}^{|T|} \times \mathbb{R}^{q} \times \mathbb{R}^{l} \times$ $\mathbb{R}^{l}$ with $\sum_{i \in I} \alpha_{i}=1, \lambda_{I_{+}(\bar{x})}^{H} \geq 0, \lambda_{I_{0+}(\bar{x})}^{G} \leq 0$ and $\lambda_{I_{+-}(\bar{x}) \cup I_{0-}(\bar{x})}^{G} \geq 0$, we define

$L\left(u, \alpha, \lambda^{g}, \lambda^{h}, \lambda^{G}, \lambda^{H}\right):=f(u)+\left(\sum_{t \in T} \lambda_{t} g_{t}(u)+\sum_{i \in I_{h}} \lambda_{i}^{h} h_{i}(u)-\sum_{i \in I_{l}} \lambda_{i}^{H} H_{i}(u)+\sum_{i \in I_{l}} \lambda_{i}^{G} G_{i}(u)\right) e$,

where $e:=(1, \ldots, 1) \in \mathbb{R}^{m}$. In the line of $[9,23]$, we consider the Wolfe type dual problem as follows:

$\left(\mathrm{D}_{W}(\bar{x})\right): \mathbb{R}_{+}^{m}-\max L\left(u, \alpha, \lambda^{g}, \lambda^{h}, \lambda^{G}, \lambda^{H}\right)$

s.t. $0 \in \sum_{i \in I} \alpha_{i} \partial^{C} f_{i}(u)+\sum_{t \in T} \lambda_{t}^{g} \partial^{C} g_{t}(u)+\sum_{i \in I_{h}} \lambda_{i}^{h} \partial^{C} h_{i}(u)-\sum_{i \in I_{l}} \lambda_{i}^{H} \partial^{C} H_{i}(u)+\sum_{i \in I_{l}} \lambda_{i}^{G} \partial^{C} G_{i}(u)$,

$\sum_{i \in I} \alpha_{i}=1, \lambda_{I_{+}(\bar{x})}^{H} \geq 0, \lambda_{I_{0+}(\bar{x})}^{G} \leq 0, \lambda_{I_{+-}(\bar{x}) \cup I_{0-}(\bar{x})}^{G} \geq 0$,

$\left(u, \alpha, \lambda^{g}, \lambda^{h}, \lambda^{G}, \lambda^{H}\right) \in \mathbb{R}^{n} \times \mathbb{R}_{+}^{m} \times \mathbb{R}_{+}^{|T|} \times \mathbb{R}^{q} \times \mathbb{R}^{l} \times \mathbb{R}^{l}$.

The feasible set of $\left(\mathrm{D}_{W}(\bar{x})\right)$ is defined by

$$
\begin{gathered}
\Omega_{W}(\bar{x}):=\left\{\left(u, \alpha, \lambda^{g}, \lambda^{h}, \lambda^{G}, \lambda^{H}\right) \in \mathbb{R}^{n} \times \mathbb{R}_{+}^{m} \times \mathbb{R}_{+}^{|T|} \times \mathbb{R}^{q} \times \mathbb{R}^{l} \times \mathbb{R}^{l} \mid\right. \\
\sum_{i \in I} \alpha_{i}=1, \lambda_{I_{+}(\bar{x})}^{H} \geq 0, \lambda_{I_{0+}(\bar{x})}^{G} \leq 0, \lambda_{I_{+-}(\bar{x}) \cup I_{0-}(\bar{x})}^{G} \geq 0 \\
\left.0 \in \sum_{i \in I} \alpha_{i} \partial^{C} f_{i}(u)+\sum_{t \in T} \lambda_{t}^{g} \partial^{C} g_{t}(u)+\sum_{i \in I_{h}} \lambda_{i}^{h} \partial^{C} h_{i}(u)-\sum_{i \in I_{l}} \lambda_{i}^{H} \partial^{C} H_{i}(u)+\sum_{i \in I_{l}} \lambda_{i}^{G} \partial^{C} G_{i}(u)\right\} .
\end{gathered}
$$

We designate by

$$
\operatorname{pr}_{\mathbb{R}^{n}} \Omega_{W}(\bar{x}):=\left\{u \in \mathbb{R}^{n} \mid\left(u, \alpha, \lambda^{g}, \lambda^{h}, \lambda^{G}, \lambda^{H}\right) \in \Omega_{W}(\bar{x})\right\}
$$

the projection of the set $\Omega(\bar{x})$ on $\mathbb{R}^{n}$. The other Wolfe type duality problem of $(\mathrm{P})$, which is not dependent on $\bar{x}$, is

$$
\begin{aligned}
\left(\mathrm{D}_{W}\right) & : \mathbb{R}_{+}^{m}-\max L\left(y, \alpha, \lambda^{g}, \lambda^{h}, \lambda^{G}, \lambda^{H}\right) \\
& =f(y)+\left(\sum_{t \in T} \lambda_{t} g_{t}(y)+\sum_{i \in I_{h}} \lambda_{i}^{h} h_{i}(y)-\sum_{i \in I_{l}} \lambda_{i}^{H} H_{i}(y)+\sum_{i \in I_{l}} \lambda_{i}^{G} G_{i}(y)\right) e,
\end{aligned}
$$

s.t. $\left(y, \alpha, \lambda^{g}, \lambda^{h}, \lambda^{G}, \lambda^{H}\right) \in \Omega_{W}:=\bigcap_{\bar{x} \in \Omega} \Omega_{W}(\bar{x})$.

Definition 4.2. Let $\bar{x} \in \Omega$.

(i) $\left(\bar{u}, \bar{\alpha}, \bar{\lambda}^{g}, \bar{\lambda}^{h}, \bar{\lambda}^{G}, \bar{\lambda}^{H}\right) \in \Omega_{W}(\bar{x})$ is a locally efficient solution of $\left(\mathrm{D}_{W}(\bar{x})\right)$, denoted by $\left(\bar{u}, \bar{\alpha}, \bar{\lambda}^{g}, \bar{\lambda}^{h}, \bar{\lambda}^{G}, \bar{\lambda}^{H}\right) \in \operatorname{loc} E\left(\mathrm{D}_{W}(\bar{x})\right)$, if there exists $U \in \mathscr{N}(\bar{u})$ such that there is no $\left(u, \alpha, \lambda^{g}, \lambda^{h}, \lambda^{G}, \lambda^{H}\right) \in \Omega_{W}(\bar{x}) \cap U$ satisfying

$$
L\left(\bar{u}, \bar{\alpha}, \bar{\lambda}^{g}, \bar{\lambda}^{h}, \bar{\lambda}^{G}, \bar{\lambda}^{H}\right) \preceq L\left(u, \alpha, \lambda^{g}, \lambda^{h}, \lambda^{G}, \lambda^{H}\right) .
$$


(ii) $\left(\bar{u}, \bar{\alpha}, \bar{\lambda}^{g}, \bar{\lambda}^{h}, \bar{\lambda}^{G}, \bar{\lambda}^{H}\right) \in \Omega_{W}(\bar{x})$ is a locally weakly efficient solution of $\left(\mathrm{D}_{W}(\bar{x})\right)$, denoted by $\left(\bar{u}, \bar{\alpha}, \bar{\lambda}^{g}, \bar{\lambda}^{h}, \bar{\lambda}^{G}, \bar{\lambda}^{H}\right) \in \operatorname{loc} W E\left(\mathrm{D}_{W}(\bar{x})\right)$, if there exists $U \in \mathscr{N}(\bar{u})$ such that there is no $\left(u, \alpha, \lambda^{g}, \lambda^{h}, \lambda^{G}, \lambda^{H}\right) \in \Omega_{W}(\bar{x}) \cap U$ fulfilling

$$
L\left(\bar{u}, \bar{\alpha}, \bar{\lambda}^{g}, \bar{\lambda}^{h}, \bar{\lambda}^{G}, \bar{\lambda}^{H}\right) \prec L\left(u, \alpha, \lambda^{g}, \lambda^{h}, \lambda^{G}, \lambda^{H}\right) .
$$

(iii) $\left(\bar{y}, \bar{\alpha}, \bar{\lambda}^{g}, \bar{\lambda}^{h}, \bar{\lambda}^{G}, \bar{\lambda}^{H}\right) \in \Omega_{W}$ is said to be a locally efficient solution of ( $\left.\mathrm{D}_{W}\right)$, denoted by $\left(\bar{y}, \bar{\alpha}, \bar{\lambda}^{g}, \bar{\lambda}^{h}, \bar{\lambda}^{G}, \bar{\lambda}^{H}\right) \in \operatorname{loc} E\left(\mathrm{D}_{W}\right)$, if there exists $U \in \mathscr{N}(\bar{y})$ such that such that there is no $\left(y, \alpha, \lambda^{g}, \lambda^{h}, \lambda^{G}, \lambda^{H}\right) \in \Omega_{W} \cap U$ satisfying

$$
L\left(\bar{y}, \bar{\lambda}^{g}, \bar{\lambda}^{h}, \bar{\lambda}^{G}, \bar{\lambda}^{H}\right) \preceq L\left(y, \bar{\alpha}, \lambda^{g}, \lambda^{h}, \lambda^{G}, \lambda^{H}\right) .
$$

(iv) $\left(\bar{y}, \bar{\alpha}, \bar{\lambda}^{g}, \bar{\lambda}^{h}, \bar{\lambda}^{G}, \bar{\lambda}^{H}\right) \in \Omega_{W}$ is called a locally weakly efficient solution of $\left(\mathrm{D}_{W}\right)$, denoted by $\left(\bar{y}, \bar{\alpha}, \bar{\lambda}^{g}, \bar{\lambda}^{h}, \bar{\lambda}^{G}, \bar{\lambda}^{H}\right) \in \operatorname{loc} W E\left(\mathrm{D}_{W}\right)$, if there exists $U \in \mathscr{N}(\bar{y})$ such that there is no $\left(y, \alpha, \lambda^{g}, \lambda^{h}, \lambda^{G}, \lambda^{H}\right) \in \Omega_{W} \cap U$ satisfying

$$
L\left(\bar{y}, \bar{\lambda}^{g}, \bar{\lambda}^{h}, \bar{\lambda}^{G}, \bar{\lambda}^{H}\right) \prec L\left(y, \bar{\alpha}, \lambda^{g}, \lambda^{h}, \lambda^{G}, \lambda^{H}\right) .
$$

If $U=\mathbb{R}^{n}$, the word "locally" is omitted.

The following propositions describe the weak duality relations between $(\mathrm{P})$ and the dual problems $\left(\mathrm{D}_{W}(\bar{x})\right)$ and $\left(\mathrm{D}_{W}\right)$.

Proposition 4.3. (Weak Duality) Let $x \in \Omega$ and $\left(y, \alpha, \lambda^{g}, \lambda^{h}, \lambda^{G}, \lambda^{H}\right) \in \Omega_{W}$. Suppose that $g_{t}(t \in$ $\left.I_{g}^{+}(x)\right), h_{i}\left(i \in I_{h}^{+}(x)\right),-h_{i}\left(i \in I_{h}^{-}(x)\right), H_{i}\left(i \in \hat{I}_{0}^{-}(x)\right),-H_{i}\left(i \in \hat{I}_{+}^{+}(x) \cup \hat{I}_{0}^{+}(x)\right), G_{i}\left(i \in I_{+0}^{+}(x) \cup\right.$ $\left.I_{+-}^{+}(x) \cup I_{00}^{+}(x) \cup I_{0-}^{+}(x)\right),-G_{i}\left(i \in I_{+0}^{-}(x) \cup I_{0+}^{-}(x) \cup I_{00}^{-}(x)\right)$ are $\partial^{C}$-convex at $y$ on $X_{W}:=\Omega \cup$ $\operatorname{pr}_{\mathbb{R}^{n}} \Omega_{W}$.

(i) If $f_{i}(i \in I)$ are $\partial^{C}$-convex at $y$ on $X_{W}$, then $f(x) \nprec L\left(y, \alpha, \lambda^{g}, \lambda^{h}, \lambda^{G}, \lambda^{H}\right)$.

(ii) If $f_{i}(i \in I)$ are strictly $\partial^{C}$-convex at $y$ on $X_{W}$, then $f(x) \npreceq L\left(y, \alpha, \lambda^{g}, \lambda^{h}, \lambda^{G}, \lambda^{H}\right)$.

Proof. For $x \in \Omega$ and $\left(y, \alpha, \lambda^{g}, \lambda^{h}, \lambda^{G}, \lambda^{H}\right) \in \Omega_{W}=\bigcap_{\bar{x} \in \Omega} \Omega_{W}(\bar{x})$, one obtains

$$
g_{t}(x) \leq 0(t \in T), h_{i}(x)=0\left(i \in I_{h}\right), H_{i}(x) \geq 0\left(i \in I_{l}\right), G_{i}(x) H_{i}(x) \leq 0\left(i \in I_{l}\right),
$$

and

$$
0 \in \sum_{i \in I} \alpha_{i} \partial^{C} f_{i}(y)+\sum_{t \in T} \lambda_{t}^{g} \partial^{C} g_{t}(y)+\sum_{i \in I_{h}} \lambda_{i}^{h} \partial^{C} h_{i}(y)-\sum_{i \in I_{l}} \lambda_{i}^{H} \partial^{C} H_{i}(y)+\sum_{i \in I_{l}} \lambda_{i}^{G} \partial^{C} G_{i}(y)
$$

with

$$
\sum_{i \in I} \alpha_{i}=1, \lambda_{I_{+}(x)}^{H} \geq 0, \lambda_{I_{0+}(x)}^{G} \leq 0, \lambda_{I_{+-}(x) \cup I_{0-}(x)}^{G} \geq 0 .
$$

It follows from (4.7) that there exist $\xi_{i}^{f} \in \partial^{C} f_{i}(y)(i \in I), \xi_{t}^{g} \in \partial^{C} g_{t}(y)(t \in T), \xi_{i}^{h} \in \partial^{C} h_{i}(y)(i \in$ $\left.I_{h}\right), \xi_{i}^{H} \in \partial^{C} H_{i}(y)\left(i \in I_{l}\right), \xi_{i}^{G} \in \partial^{C} G_{i}(y)\left(i \in I_{l}\right)$ such that

$$
\sum_{i \in I} \alpha_{i} \xi_{i}^{f}+\sum_{t \in J} \lambda_{t}^{g} \xi_{t}^{g}+\sum_{i \in I_{h}} \lambda_{i}^{h} \xi_{i}^{h}-\sum_{i \in I_{l}} \lambda_{i}^{H} \xi_{i}^{H}+\sum_{i \in I_{l}} \lambda_{i}^{G} \xi_{i}^{G}=0 .
$$

Therefore, we conclude from (4.6), the $\partial^{C}$-convexity of $g_{t}\left(t \in I_{g}^{+}(x)\right), h_{i}\left(i \in I_{h}^{+}(x)\right),-h_{i}(i \in$ $\left.I_{h}^{-}(x)\right), H_{i}\left(i \in \hat{I}_{0}^{-}(x)\right),-H_{i}\left(i \in \hat{I}_{+}^{+}(x) \cup \hat{I}_{0}^{+}(x)\right), G_{i}\left(i \in I_{+0}^{+}(x) \cup I_{+-}^{+}(x) \cup I_{00}^{+}(x) \cup I_{0-}^{+}(x)\right),-G_{i}(i \in$ $\left.I_{+0}^{-}(x) \cup I_{0+}^{-}(x) \cup I_{00}^{-}(x)\right)$ at $y$ on $X_{W}$ and the definitions of the index sets that

$$
g_{t}(y)+\left\langle\xi_{t}^{g}, x-y\right\rangle \leq g_{t}(x) \leq 0, \lambda_{t}^{g}>0, \forall t \in I_{g}^{+}(x),
$$




$$
\begin{gathered}
h_{i}(y)+\left\langle\xi_{i}^{h}, x-y\right\rangle \leq h_{i}(x)=0, \lambda_{i}^{h}>0, \forall i \in I_{h}^{+}(x), \\
-h_{i}(y)+\left\langle-\xi_{i}^{h}, x-y\right\rangle \leq-h_{i}(x)=0, \lambda_{i}^{h}<0, \forall i \in I_{h}^{-}(x), \\
H_{i}(y)+\left\langle\xi_{i}^{H}, x-y\right\rangle \leq H_{i}(x)=0, \lambda_{i}^{H}<0, \forall i \in \hat{I}_{0}^{-}(x), \\
-H_{i}(y)+\left\langle-\xi_{i}^{H}, x-y\right\rangle \leq-H_{i}(x)<0, \lambda_{i}^{H}>0, \forall i \in \hat{I}_{+}^{+}(x), \\
-H_{i}(y)+\left\langle-\xi_{i}^{H}, x-y\right\rangle \leq-H_{i}(x)=0, \lambda_{i}^{H}>0, \forall i \in \hat{I}_{0}^{+}(x), \\
G_{i}(y)+\left\langle\xi_{i}^{G}, x-y\right\rangle \leq G_{i}(x)=0, \lambda_{i}^{G}>0, \forall i \in I_{+0}^{+}(x) \cup I_{00}^{+}(x), \\
G_{i}(y)+\left\langle\xi_{i}^{G}, x-y\right\rangle \leq G_{i}(x)<0, \lambda_{i}^{G}>0, \forall i \in I_{+-}^{+}(x) \cup I_{0-}^{+}(x), \\
-G_{i}(y)+\left\langle-\xi_{i}^{G}, x-y\right\rangle \leq-G_{i}(x)=0, \lambda_{i}^{G}<0, \forall i \in I_{+0}^{-}(x) \cup I_{00}^{-}(x), \\
-G_{i}(y)+\left\langle-\xi_{i}^{G}, x-y\right\rangle \leq-G_{i}(x)<0, \lambda_{i}^{G}<0, \forall i \in I_{0+}^{-}(x) .
\end{gathered}
$$

The above inequalities imply that

$$
\begin{aligned}
& \sum_{t \in T} \lambda_{t} g_{t}(y)+\sum_{i \in I_{h}} \lambda_{i}^{h} h_{i}(y)-\sum_{i \in I_{l}} \lambda_{i}^{H} H_{i}(y)+\sum_{i \in I_{l}} \lambda_{i}^{G} G_{i}(y) \\
& \quad+\left\langle\sum_{t \in T} \lambda_{t}^{g} \xi_{t}^{g}+\sum_{i \in I_{h}} \lambda_{i}^{h} \xi_{i}^{h}-\sum_{i \in I_{l}} \lambda_{i}^{H} \xi_{i}^{H}+\sum_{i \in I_{l}} \lambda_{i}^{G} \xi_{i}^{G}, x-y\right\rangle \leq 0 .
\end{aligned}
$$

This together with (4.9) proves that

$$
\begin{aligned}
\left\langle\sum_{i \in I} \alpha_{i} \xi_{i}^{f}, x-y\right\rangle & =-\left\langle\sum_{t \in T} \lambda_{t}^{g} \xi_{t}^{g}+\sum_{i \in I_{h}} \lambda_{i}^{h} \xi_{i}^{h}-\sum_{i \in I_{l}} \lambda_{i}^{H} \xi_{i}^{H}+\sum_{i \in I_{l}} \lambda_{i}^{G} \xi_{i}^{G}, x-y\right\rangle \\
& \geq \sum_{t \in T} \lambda_{t} g_{t}(y)+\sum_{i \in I_{h}} \lambda_{i}^{h} h_{i}(y)-\sum_{i \in I_{l}} \lambda_{i}^{H} H_{i}(y)+\sum_{i \in I_{l}} \lambda_{i}^{G} G_{i}(y) .
\end{aligned}
$$

(i) Suppose that

$$
f(x) \prec L\left(y, \alpha, \lambda^{g}, \lambda^{h}, \lambda^{G}, \lambda^{H}\right) .
$$

Then, we deduce from (4.11) and $\alpha \in \mathbb{R}_{+}^{m}$ that $\left\langle\alpha, f(x)-L\left(y, \alpha, \lambda^{g}, \lambda^{h}, \lambda^{G}, \lambda^{H}\right)\right\rangle<0$, which is equivalent to

$$
\sum_{i=1}^{m} \alpha_{i}\left(f_{i}(x)-f_{i}(y)\right)-\sum_{i=1}^{m} \alpha_{i}\left(\sum_{t \in T} \lambda_{t} g_{t}(y)+\sum_{i \in I_{h}} \lambda_{i}^{h} h_{i}(y)-\sum_{i \in I_{l}} \lambda_{i}^{H} H_{i}(y)+\sum_{i \in I_{l}} \lambda_{i}^{G} G_{i}(y)\right)<0 .
$$

The above inequality together with $\sum_{i=1}^{m} \alpha_{i}=1$ yields that

$$
\sum_{i=1}^{m} \alpha_{i}\left(f_{i}(x)-f_{i}(y)\right)<\sum_{t \in T} \lambda_{t} g_{t}(y)+\sum_{i \in I_{h}} \lambda_{i}^{h} h_{i}(y)-\sum_{i \in I_{l}} \lambda_{i}^{H} H_{i}(y)+\sum_{i \in I_{l}} \lambda_{i}^{G} G_{i}(y) .
$$

The $\partial^{C}$-convexity of $f_{i}(i \in I)$ at $y$ on $X_{W}$ confirms that $\left\langle\xi_{i}^{f}, x-y\right\rangle \leq f_{i}(x)-f_{i}(y), \forall i \in I$, which leads that

$$
\left\langle\sum_{i=1}^{m} \alpha_{i} \xi_{i}^{f}, x-y\right\rangle \leq \sum_{i=1}^{m} \alpha_{i}\left(f_{i}(x)-f_{i}(y)\right)
$$

We verify from (4.12) and (4.13) that

$$
\left\langle\sum_{i=1}^{m} \alpha_{i} \xi_{i}^{f}, x-y\right\rangle<\left(\sum_{t \in T} \lambda_{t} g_{t}(y)+\sum_{i \in I_{h}} \lambda_{i}^{h} h_{i}(y)-\sum_{i \in I_{l}} \lambda_{i}^{H} H_{i}(y)+\sum_{i \in I_{l}} \lambda_{i}^{G} G_{i}(y)\right),
$$


which contradicts (4.10).

(ii) Assume that

$$
f(x) \preceq L\left(y, \alpha, \lambda^{g}, \lambda^{h}, \lambda^{G}, \lambda^{H}\right),
$$

We claim that $x \neq y$. If otherwise, we use (4.14) and $x=y$ to derive that

$$
a:=-\left(\sum_{t \in T} \lambda_{t} g_{t}(y)+\sum_{i \in I_{h}} \lambda_{i}^{h} h_{i}(y)-\sum_{i \in I_{l}} \lambda_{i}^{H} H_{i}(y)+\sum_{i \in I_{l}} \lambda_{i}^{G} G_{i}(y)\right) e \preceq 0 .
$$

Observe by $y=x \in \Omega$ and (4.8) that

$$
\begin{gathered}
g_{t}(y)=g_{t}(x) \leq 0, \forall t \in T \text { and } \lambda \in \mathbb{R}_{+}^{|T|}, \\
h_{i}(y)=h_{i}(x)=0, \forall i \in I_{h}, \lambda_{i}^{h} \in \mathbb{R}, i \in I_{h}, \\
H_{i}(y)=H_{i}(x) \geq 0, \forall i \in I_{+}(x), \lambda_{I_{+}(x)}^{H} \geq 0, \\
H_{i}(y)=H_{i}(x)=0, \forall i \in I_{0}(x), \lambda_{i}^{H} \in \mathbb{R}, \forall i \in I_{0}(x), \\
G_{i}(y)=G_{i}(x)=0, \forall i \in I_{+0}(x) \cup I_{00}(x), \lambda_{i}^{G} \in \mathbb{R}, \forall i \in I_{+0}(x) \cup I_{00}(x), \\
G_{i}(y)=G_{i}(x)>0, \forall i \in I_{0+}(x), \lambda_{I_{0+}(x)}^{G} \leq 0,
\end{gathered}
$$

and

$$
G_{i}(y)=G_{i}(x)<0, \forall i \in I_{+-}(x) \cup I_{0-}(x), \lambda_{I_{+-}(x) \cup I_{0-}(x)} \geq 0 .
$$

The above inequalities leads that

$$
\sum_{t \in T} \lambda_{t} g_{t}(y)+\sum_{i \in I_{h}} \lambda_{i}^{h} h_{i}(y)-\sum_{i \in I_{l}} \lambda_{i}^{H} H_{i}(y)+\sum_{i \in I_{l}} \lambda_{i}^{G} G_{i}(y) \leq 0 .
$$

Hence, $a_{i} \geq 0, \forall i \in I$, contradicts (4.15), which in turn implies $x \neq y$. On the other hand, we deduce from (4.14) and $\alpha \in \mathbb{R}_{+}^{m}$ that $\left\langle\alpha, f(x)-L\left(y, \alpha, \lambda^{g}, \lambda^{h}, \lambda^{G}, \lambda^{H}\right)\right\rangle \leq 0$. In other words,

$$
\sum_{i=1}^{m} \alpha_{i}\left(f_{i}(x)-f_{i}(y)\right)-\sum_{i=1}^{m} \alpha_{i}\left(\sum_{t \in T} \lambda_{t} g_{t}(y)+\sum_{i \in I_{h}} \lambda_{i}^{h} h_{i}(y)-\sum_{i \in I_{l}} \lambda_{i}^{H} H_{i}(y)+\sum_{i \in I_{l}} \lambda_{i}^{G} G_{i}(y)\right) \leq 0 .
$$

Employing this together with $\sum_{i=1}^{m} \alpha_{i}=1$ gives us the inequality

$$
\sum_{i=1}^{m} \alpha_{i}\left(f_{i}(x)-f_{i}(y)\right) \leq \sum_{t \in T} \lambda_{t} g_{t}(y)+\sum_{i \in I_{h}} \lambda_{i}^{h} h_{i}(y)-\sum_{i \in I_{l}} \lambda_{i}^{H} H_{i}(y)+\sum_{i \in I_{l}} \lambda_{i}^{G} G_{i}(y) .
$$

Since $f_{i}(i \in I)$ are strictly $\partial^{C}$-convex at $y$ on $X_{W}$ and $x \neq y$, we have

$$
\left\langle\xi_{i}^{f}, x-y\right\rangle<f_{i}(x)-f_{i}(y), \quad \forall i \in I
$$

which implies that

$$
\left\langle\sum_{i=1}^{m} \alpha_{i} \xi_{i}^{f}, x-y\right\rangle<\sum_{i=1}^{m} \alpha_{i}\left(f_{i}(x)-f_{i}(y)\right) .
$$

It follows from (4.16) and (4.17) that

$$
\left\langle\sum_{i=1}^{m} \alpha_{i} \xi_{i}^{f}(y), x-y\right\rangle<\sum_{t \in T} \lambda_{t} g_{t}(y)+\sum_{i \in I_{h}} \lambda_{i}^{h} h_{i}(y)-\sum_{i \in I_{l}} \lambda_{i}^{H} H_{i}(y)+\sum_{i \in I_{l}} \lambda_{i}^{G} G_{i}(y),
$$

which contradicts (4.10). 
Corollary 4.2. (Weak Duality) Let $\bar{x} \in \Omega$ and $\left(u, \alpha, \lambda^{g}, \lambda^{h}, \lambda^{G}, \lambda^{H}\right) \in \Omega_{W}(\bar{x})$. Suppose that $g_{t}\left(t \in I_{g}^{+}(\bar{x})\right), h_{i}\left(i \in I_{h}^{+}(\bar{x})\right),-h_{i}\left(i \in I_{h}^{-}(\bar{x})\right), H_{i}\left(i \in \hat{I}_{0}^{-}(\bar{x})\right),-H_{i}\left(i \in \hat{I}_{+}^{+}(\bar{x}) \cup \hat{I}_{0}^{+}(\bar{x})\right), G_{i}\left(i \in I_{+0}^{+}(\bar{x}) \cup\right.$ $\left.I_{+-}^{+}(\bar{x}) \cup I_{00}^{+}(\bar{x}) \cup I_{0-}^{+}(\bar{x})\right),-G_{i}\left(i \in I_{+0}^{-}(\bar{x}) \cup I_{0+}^{-}(\bar{x}) \cup I_{00}^{-}(\bar{x})\right)$ are $\partial^{C}$-convex at $u$ on $\bar{X}_{W}:=\Omega \cup$ $\Omega_{W}(\bar{x})$.

(i) If $f_{i}(i \in I)$ are $\partial^{C}$-convex at $u$ on $\bar{X}_{W}$, then $f(\bar{x}) \nprec L\left(u, \alpha, \lambda^{g}, \lambda^{h}, \lambda^{G}, \lambda^{H}\right)$.

(ii) If $f_{i}(i \in I)$ are strictly $\partial^{C}$-convex at $u$ on $\bar{X}_{W}$, then $f(\bar{x}) \npreceq L\left(u, \alpha, \lambda^{g}, \lambda^{h}, \lambda^{G}, \lambda^{H}\right)$.

Proposition 4.4. (Strong Duality) Let $\bar{x} \in \Omega$ be a locally weakly efficient solution of $(P)$. If (VC$A C Q)$ holds at $\bar{x}$ and the set $\Delta_{1}$ is closed, then there exists $\left(\bar{\alpha}, \bar{\lambda}^{g}, \bar{\lambda}^{h}, \bar{\lambda}^{G}, \bar{\lambda}^{H}\right) \in \mathbb{R}_{+}^{m} \times \mathbb{R}_{+}^{|T|} \times$ $\mathbb{R}^{q} \times \mathbb{R}^{l} \times \mathbb{R}^{l}$ with $\bar{\lambda}_{I_{+}(\bar{x})}^{H}=0, \bar{\lambda}_{I_{00}(\bar{x}) \cup I_{0-}(\bar{x})}^{H} \geq 0, \bar{\lambda}_{I_{+-}(\bar{x}) \cup I_{0+}(\bar{x}) \cup I_{0_{-}(\bar{x})}}^{G}=0$ and $\bar{\lambda}_{I_{+0}(\bar{x}) \cup I_{00}(\bar{x})} \geq 0$ such that $\left(\bar{x}, \bar{\alpha}, \bar{\lambda}^{g}, \bar{\lambda}^{h}, \bar{\lambda}^{G}, \bar{\lambda}^{H}\right) \in \Omega_{W}(\bar{x})$ and $f(\bar{x})=L\left(\bar{x}, \bar{\alpha}, \bar{\lambda}^{g}, \bar{\lambda}^{h}, \bar{\lambda}^{G}, \bar{\lambda}^{H}\right)$. Assume further that $g_{t}\left(t \in I_{g}^{+}(\bar{x})\right), h_{i}\left(i \in I_{h}^{+}(\bar{x})\right),-h_{i}\left(i \in I_{h}^{-}(\bar{x})\right), H_{i}\left(i \in \hat{I}_{0}^{-}(\bar{x})\right),-H_{i}\left(i \in \hat{I}_{+}^{+}(\bar{x}) \cup \hat{I}_{0}^{+}(\bar{x})\right), G_{i}(i \in$ $\left.I_{+0}^{+}(\bar{x}) \cup I_{+-}^{+}(\bar{x}) \cup I_{00}^{+}(\bar{x}) \cup I_{0-}^{+}(\bar{x})\right),-G_{i}\left(i \in I_{+0}^{-}(\bar{x}) \cup I_{0+}^{-}(\bar{x}) \cup I_{00}^{-}(\bar{x})\right)$ are $\partial^{C}$-convex at $\bar{x}$ on $\bar{X}_{W}$.

(i) If $f_{i}(i \in I)$ are $\partial^{C}$-convex at $\bar{x}$ on $\bar{X}_{W}$, then $\left(\bar{x}, \bar{\alpha}, \bar{\lambda}^{g}, \bar{\lambda}^{h}, \bar{\lambda}^{G}, \bar{\lambda}^{H}\right)$ is a weakly efficient solution of $D_{W}(\bar{x})$.

(ii) If $f_{i}(i \in I)$ are strictly $\partial^{C}$-convex at $\bar{x}$ on $\bar{X}_{W}$, then $\left(\bar{x}, \bar{\alpha}, \bar{\lambda}^{g}, \bar{\lambda}^{h}, \bar{\lambda}^{G}, \bar{\lambda}^{H}\right)$ is an efficient solution of $D_{W}(\bar{x})$.

Proof. In view of Proposition 3.1 (ii), there exists $\left(\bar{\alpha}, \bar{\lambda}^{g}, \bar{\lambda}^{h}, \bar{\lambda}^{G}, \bar{\lambda}^{H}\right) \in \mathbb{R}_{+}^{m} \times \Lambda(\bar{x}) \times \mathbb{R}^{q} \times \mathbb{R}^{l} \times$ $\mathbb{R}^{l}$ with $\bar{\lambda}_{I_{+}(\bar{x})}^{H}=0, \bar{\lambda}_{I_{00}(\bar{x}) \cup I_{0-}(\bar{x})}^{H} \geq 0, \bar{\lambda}_{I_{+-}(\bar{x}) \cup I_{0+}(\bar{x}) \cup I_{0_{-}(\bar{x})}}^{G}=0$ and $\bar{\lambda}_{I_{+0}(\bar{x}) \cup I_{00}(\bar{x})}^{G} \geq 0$ such that

$$
0 \in \sum_{i \in I} \bar{\alpha}_{i} \partial^{C} f_{i}(\bar{x})+\sum_{t \in T} \bar{\lambda}_{t}^{g} \partial^{C} g_{t}(\bar{x})+\sum_{i \in I_{h}} \bar{\lambda}_{i}^{h} \partial^{C} h_{i}(\bar{x})-\sum_{i \in I_{l}} \bar{\lambda}_{i}^{H} \partial^{C} H_{i}(\bar{x})+\sum_{i \in I_{l}} \bar{\lambda}_{i}^{G} \partial^{C} G_{i}(\bar{x}) .
$$

Since $\bar{\lambda}^{g} \in \Lambda(\bar{x})$, one has $\bar{\lambda}_{t}^{g} g_{t}(\bar{x})=0$ for all $t \in T$, and thus, $\sum_{t \in T} \bar{\lambda}_{t}^{g} g_{t}(\bar{x})=0$. The fact that $\bar{x} \in \Omega$ guarantees that $\sum_{i \in I_{h}} \bar{\lambda}_{i}^{h} h_{i}(\bar{x})=0$. Moreover, we observe by $\bar{\lambda}_{I_{+}(\bar{x})}^{H}=0$ and $H_{i}(\bar{x})=0$ for all $i \in I_{0}(\bar{x})$ that $\sum_{i \in I_{l}} \bar{\lambda}_{i}^{H} H_{i}(\bar{x})=0$. Analogously, as $\bar{\lambda}_{I_{+-}}^{G}(\bar{x}) \cup I_{0+}(\bar{x}) \cup I_{0-}(\bar{x})=0$ and $G_{i}(\bar{x})=0$ for all $i \in I_{+0}(\bar{x}) \cup I_{00}(\bar{x})$, we know that $\sum_{i \in I_{l}} \bar{\lambda}_{i}^{G} G_{i}(\bar{x})=0$. Thus, $\left(\bar{x}, \bar{\alpha}, \bar{\lambda}^{g}, \bar{\lambda}^{h}, \bar{\lambda}^{G}, \bar{\lambda}^{H}\right) \in \Omega_{W}(\bar{x})$ and

$$
\sum_{t \in T} \lambda_{t} g_{t}(\bar{x})+\sum_{i \in I_{h}} \lambda_{i}^{h} h_{i}(\bar{x})-\sum_{i \in I_{l}} \lambda_{i}^{H} H_{i}(\bar{x})+\sum_{i \in I_{l}} \lambda_{i}^{G} G_{i}(\bar{x})=0
$$

which is nothing else but the following equality $f(\bar{x})=L\left(\bar{x}, \bar{\alpha}, \bar{\lambda}^{g}, \bar{\lambda}^{h}, \bar{\lambda}^{G}, \bar{\lambda}^{H}\right)$.

(i) Now, arguing by contradiction, let us suppose that $\left(\bar{x}, \bar{\alpha}, \bar{\lambda}^{g}, \bar{\lambda}^{h}, \bar{\lambda}^{G}, \bar{\lambda}^{H}\right)$ is not a weakly efficient solution of $D_{W}(\bar{x})$. By definition, there exists $\left(u, \alpha, \lambda^{g}, \lambda^{h}, \lambda^{G}, \lambda^{H}\right) \in \Omega_{W}(\bar{x})$ such that

$$
L\left(\bar{x}, \bar{\alpha}, \bar{\lambda}^{g}, \bar{\lambda}^{h}, \bar{\lambda}^{G}, \bar{\lambda}^{H}\right) \prec L\left(u, \alpha, \lambda^{g}, \lambda^{h}, \lambda^{G}, \lambda^{H}\right) .
$$

This shows that

$$
f(\bar{x}) \prec L\left(u, \alpha, \lambda^{g}, \lambda^{h}, \lambda^{G}, \lambda^{H}\right) .
$$

which contradicts Corollary 4.2 (i). So, $\left(\bar{x}, \bar{\alpha}, \bar{\lambda}^{g}, \bar{\lambda}^{h}, \bar{\lambda}^{G}, \bar{\lambda}^{H}\right)$ is a weakly efficient solution of $\left(D_{W}(\bar{x})\right)$.

(ii) Reasoning to the contrary, let us assume that $\left(\bar{x}, \bar{\alpha}, \bar{\lambda}^{g}, \bar{\lambda}^{h}, \bar{\lambda}^{G}, \bar{\lambda}^{H}\right)$ is not an efficient solution of $D_{W}(\bar{x})$. Then, it guarantees the existence of $\left(u, \alpha, \lambda^{g}, \lambda^{h}, \lambda^{G}, \lambda^{H}\right) \in \Omega_{W}(\bar{x})$ such that

$$
L\left(\bar{x}, \bar{\alpha}, \bar{\lambda}^{g}, \bar{\lambda}^{h}, \bar{\lambda}^{G}, \bar{\lambda}^{H}\right) \preceq L\left(u, \alpha, \lambda^{g}, \lambda^{h}, \lambda^{G}, \lambda^{H}\right),
$$


Consequently,

$$
f(\bar{x}) \preceq L\left(u, \alpha, \lambda^{g}, \lambda^{h}, \lambda^{G}, \lambda^{H}\right) .
$$

which contradicts Corollary 4.2 (ii). So, $\left(\bar{x}, \bar{\alpha}, \bar{\lambda}^{g}, \bar{\lambda}^{h}, \bar{\lambda}^{G}, \bar{\lambda}^{H}\right)$ is an efficient solution of $\left(D_{W}(\bar{x})\right)$.

Example 4.1. Let $m=n=2$ and $l=1$. Consider the following $(\mathrm{P})$ :

$$
\begin{gathered}
\mathbb{R}_{+}^{2}-\min \quad f(x)=\left(x_{1}^{2}+x_{2}^{2}+2 x_{2},\left|x_{1}\right|+\left|x_{2}\right|\right), \\
\text { s.t. } \quad g_{t}(x)=-t x_{1} \leq 0, t \in T=\mathbb{N}, \\
H_{1}(x)=x_{1}+2 x_{2} \geq 0, \\
G_{1}(x) H_{1}(x)=x_{1}\left(x_{1}+2 x_{2}\right) \leq 0 .
\end{gathered}
$$

Then, $\Omega=\left\{x \in \mathbb{R}^{2} \mid x_{1}>0, x_{1}+2 x_{2}=0\right\} \cup\left\{x \in \mathbb{R}^{2} \mid x_{1}=0, x_{2} \geq 0\right\}$. Moreover,

$$
\begin{gathered}
\partial^{C} f_{1}(x)=\left\{\left(2 x_{1}, 2 x_{2}+2\right)\right\}, \partial^{C} f_{2}(x)=\operatorname{co}\{(1, \pm 1),(-1, \pm 1)\}=[-1,1] \times[-1,1], \\
\partial^{C} g_{t}(x)=\{(-t, 0)\}, t \in T, \partial^{C} G_{1}(\bar{x})=\{(1,0)\}, \partial^{C} H_{1}(\bar{x})=\{(1,2)\} .
\end{gathered}
$$

For any $\bar{x} \in \Omega$,

$$
\begin{aligned}
\left(\mathrm{D}_{M W}(\bar{x})\right) & : \mathbb{R}_{+}^{2}-\max L\left(u, \alpha, \lambda^{g}, \lambda^{G}, \lambda^{H}\right) \\
& =\left(u_{1}^{2}+u_{2}^{2}+2 u_{1},\left|u_{1}\right|+\left|u_{2}\right|\right)+\left(\sum_{t \in T}\left(-t u_{1}\right)-\lambda_{1}^{H}\left(u_{1}+2 u_{2}\right)+\lambda_{1}^{G} u_{1}\right)
\end{aligned}
$$

s.t. $(0,0) \in \alpha_{1}\left(2 u_{1}, 2 u_{2}+2\right)+\alpha_{2} \cdot[-1,1] \times[-1,1]+\sum_{t \in T} \lambda_{t}^{g}(-t, 0)-\lambda_{1}^{H}(1,2)+\lambda_{1}^{G}(1,0)$,

$$
\alpha_{1}+\alpha_{2}=1, \lambda_{1}^{H} \begin{cases}\geq 0, & \text { if } 1 \in I_{+}(\bar{x}), \quad \lambda_{1}^{G}\left\{\begin{array}{ll}
\leq 0, & \text { if } 1 \in I_{0+}(\bar{x}), \\
\in 0, & \text { if } 1 \in I_{+-}(\bar{x}) \cup I_{0-}(\bar{x}), \\
\in \mathbb{R}, & \text { if } 1 \in I_{0}(\bar{x}),
\end{array} \quad \text { if } 1 \in I_{+0}(\bar{x}) \cup I_{00}(\bar{x}),\right.\end{cases}
$$

$\left(u, \alpha, \lambda^{g}, \lambda_{1}^{G}, \lambda_{1}^{H}\right) \in \mathbb{R}^{2} \times \mathbb{R}_{+}^{2} \times \mathbb{R}_{+}^{|T|} \times \mathbb{R} \times \mathbb{R}$.

Taking $\bar{x}=(0,0) \in \Omega$, one has

$$
\begin{gathered}
\mathscr{T}(\Omega, \bar{x})=\Omega, \partial^{C} f_{1}(\bar{x})=\{(0,2)\}, \partial^{C} f_{2}(\bar{x})=[-1,1] \times[-1,1], \\
I_{g}=\mathbb{N}, \partial^{C} g_{t}(\bar{x})=\{(-t, 0)\}, t \in T,\left(\bigcup_{t \in I_{g}} \partial^{C} g_{t}(\bar{x})\right)^{-}=\left\{x \in \mathbb{R}^{2} \mid x_{1} \geq 0\right\}, \\
I_{+}=I_{0+}=I_{0-}=\emptyset, I_{00}=\{1\}, \partial^{C} G_{1}(\bar{x})=\{(1,0)\}, \partial^{C} H_{1}(\bar{x})=\{(1,2)\}, \\
\left(\bigcup_{i \in I_{00}}\left(-\partial^{C} H_{i}(\bar{x})\right)\right)^{-}=\left\{x \in \mathbb{R}^{2} \mid x_{1}+2 x_{2} \geq 0\right\},\left(\bigcup_{i \in I_{00}} \partial^{C} G_{i}(\bar{x})\right)^{-}=\left\{x \in \mathbb{R}^{2} \mid x_{1} \leq 0\right\},
\end{gathered}
$$

and

$$
\left(\bigcup_{t \in I_{g}} \partial^{C} g_{t}(\bar{x})\right)^{-} \cap\left(\bigcup_{i \in I_{00}}\left(-\partial^{C} H_{i}(\bar{x})\right)\right)^{-} \cap\left(\bigcup_{i \in I_{00}} \partial^{C} G_{i}(\bar{x})\right)^{-}=\left\{x \in \mathbb{R}^{2} \mid x_{1}=0, x_{2} \geq 0\right\} .
$$

Hence,

$$
\left(\bigcup_{t \in I_{g}} \partial^{C} g_{t}(\bar{x})\right)^{-} \cap\left(\bigcup_{i \in I_{00}}\left(-\partial^{C} H_{i}(\bar{x})\right)\right)^{-} \cap\left(\bigcup_{i \in I_{00}} \partial^{C} G_{i}(\bar{x})\right)^{-} \subset \mathscr{T}(\Omega, \bar{x}),
$$

leading that (VC-ACQ) satisfies at $\bar{x}$. Moreover,

$$
\Delta_{1}=\operatorname{pos}\left(\bigcup_{t \in I_{g}} \partial^{C} g_{t}(\bar{x}) \cup \bigcup_{i \in I_{00}}\left(-\partial^{C} H_{i}(\bar{x})\right) \cup \bigcup_{i \in I_{00}} \partial^{C} G_{i}(\bar{x})\right)=\left\{x \in \mathbb{R}^{2} \mid x_{2} \leq 0\right\}
$$


is closed. Notice that

$$
\begin{aligned}
& f_{1}(x)= \begin{cases}\frac{5}{4} x_{1}^{2}-x_{1} \leq 0=f_{1}(\bar{x}), & \text { if } x \in\left\{x \in \mathbb{R}^{2} \mid \frac{4}{5} \geq x_{1} \geq 0, x_{1}+2 x_{2}=0\right\}, \\
x_{2}^{2}+2 x_{2} \geq 0=f_{1}(\bar{x}), & \text { if } x \in\left\{x \in \mathbb{R}^{2} \mid x_{1}=0, x_{2} \geq 0\right\} .\end{cases} \\
& f_{2}(x) \geq f_{2}(\bar{x}), \forall x \in \Omega,
\end{aligned}
$$

Thus, $\bar{x} \in W E(P)$, but $\bar{x} \notin E(P)$. Hence, all the hypotheses of Proposition 4.4 (i) are fulfilled. Now, if we select $\bar{\alpha}_{1}=\frac{1}{4}, \bar{\alpha}_{2}=\frac{3}{4}, \bar{\lambda}_{1}^{H}=\frac{1}{4}, \bar{\lambda}_{1}^{G}=0$ and

$$
\bar{\lambda}^{g}(t)= \begin{cases}\frac{1}{2}, & \text { if } t=1, \\ 0, & \text { otherwise, }\end{cases}
$$

then

$$
\begin{aligned}
(0,0) & =\frac{1}{4}(0,2)+\frac{3}{4}(1,0)+\frac{1}{2} \cdot(-1,0)-\frac{1}{4} \cdot(1,2)+0 \cdot(1,0) \\
& \in \frac{1}{4}(0,2)+\frac{3}{4} \cdot[-1,1] \times[-1,1]+\sum_{t \in T} \lambda_{t}^{g}(-t, 0)-\frac{1}{4} \cdot \lambda_{1}^{H}(0,1)+\lambda_{1}^{G} \cdot[-1,1] \times\{0\},
\end{aligned}
$$

and,

$$
I_{+}(\bar{x})=I_{0+}(\bar{x})=I_{0-}(\bar{x})=\emptyset, I_{00}(\bar{x})=\{1\}, \bar{\lambda}_{1}^{H}=\frac{1}{4}, \bar{\lambda}_{1}^{G}=0,1 \in I_{00}(\bar{x}),
$$

which gives the result $\left(\bar{x}, \bar{\alpha}, \bar{\lambda}^{g}, \bar{\lambda}_{1}^{G}, \bar{\lambda}_{1}^{H}\right) \in \Omega_{W}(\bar{x})$ and $f(\bar{x})=L\left(\bar{x}, \bar{\alpha}, \bar{\lambda}^{g}, \bar{\lambda}_{1}^{G}, \bar{\lambda}_{1}^{H}\right)$. Note that, for the above $\left(\bar{x}, \bar{\alpha}, \bar{\lambda}^{g}, \bar{\lambda}_{1}^{G}, \bar{\lambda}_{1}^{H}\right), \hat{I}_{00}^{+}(\bar{x})=\{1\}, \hat{I}_{00}^{-}(\bar{x})=I_{00}^{+}(\bar{x})=I_{00}^{-}(\bar{x})=\emptyset$. Moreover, we can verify that $f_{1}, f_{2}, g_{t}\left(t \in I_{g}^{+}(\bar{x})\right),-H_{i}\left(i \in \hat{I}_{00}^{+}(\bar{x})\right)$ are $\partial^{C}$-convex at $\bar{x}$ on $\mathbb{R}^{2}$. Hence, Proposition 4.4 (i) asserts that $\left(\bar{x}, \bar{\alpha}, \bar{\lambda}^{g}, \bar{\lambda}_{1}^{G}, \bar{\lambda}_{1}^{H}\right)$ is a weakly efficient solution of $\left(\mathrm{D}_{W}(\bar{x})\right)$.

We can check directly that $\left(\bar{x}, \bar{\alpha}, \bar{\lambda}^{g}, \bar{\lambda}_{1}^{G}, \bar{\lambda}_{1}^{H}\right)$ is a weakly efficient solution of $\left(\mathrm{D}_{W}(\bar{x})\right)$ as follows. Firstly, we conclude from $\bar{x}=(0,0)$ and $I_{+}(\bar{x})=I_{0+}(\bar{x})=I_{0-}=\emptyset, I_{00}(\bar{x})=\{1\}$ that

$$
\begin{gathered}
\Omega_{W}(\bar{x})=\left\{\left(u, \alpha, \lambda^{g}, \lambda_{1}^{G}, \lambda_{1}^{H}\right) \in \mathbb{R}_{+}^{2} \times \mathbb{R}^{2} \times \mathbb{R}_{+}^{|T|} \times \mathbb{R} \times \mathbb{R} \mid \alpha_{1}+\alpha_{2}=1, \lambda_{1}^{H} \in \mathbb{R}, \lambda_{1}^{G} \in \mathbb{R},\right. \\
\left.(0,0) \in \alpha_{1}\left(2 u_{1}, 2 u_{2}+2\right)+\alpha_{2} \cdot[-1,1] \times[-1,1]+\sum_{t \in T} \lambda_{t}^{g}(-t, 0)-\lambda_{1}^{H}(1,2)+\lambda_{1}^{G}(1,0)\right\} .
\end{gathered}
$$

Now, for an arbitrary $u \in \Omega_{W}(\bar{x})$, the $\partial^{C}$-convexity of $g_{t}\left(t \in I_{g}^{+}(\bar{x})\right), H_{i}\left(i \in \hat{I}_{00}^{-}(\bar{x})\right),-H_{i}(i \in$ $\left.\hat{I}_{00}^{+}(\bar{x})\right), G_{i}\left(i \in I_{00}^{+}\right),-G_{i}\left(i \in I_{00}^{-}\right)$at $u$ on $\mathbb{R}^{2}$ and the definitions of the index sets leads us to the inequalities

$$
\begin{gathered}
g_{t}(u)+\langle(-t, 0), \bar{x}-u\rangle \leq g_{t}(\bar{x})=0, \lambda_{t}^{g}>0, \forall t \in I_{g}^{+}(\bar{x}), \\
H_{1}(u)+\langle(1,1), \bar{x}-u\rangle \leq H_{1}(\bar{x})=0, \lambda_{1}^{H}<0, \text { if } 1 \in \hat{I}_{00}^{-}(\bar{x}), \\
-H_{1}(u)+\langle-(1,1), \bar{x}-u\rangle \leq-H_{1}(\bar{x})=0, \lambda_{i}^{H}>0, \text { if } 1 \in \hat{I}_{00}^{+}(\bar{x}), \\
G_{1}(u)+\langle(1,0), \bar{x}-u\rangle \leq G_{1}(\bar{x})=0, \lambda_{1}^{G}>0, \text { if } 1 \in I_{00}^{+}(\bar{x}),
\end{gathered}
$$

and

$$
-G_{1}(u)+\langle-(1,0), \bar{x}-u\rangle \leq-G_{1}(\bar{x})=0, \lambda_{1}^{G}<0 \text {, if } 1 \in I_{00}^{-}(\bar{x}) .
$$

We deduce from the above inequalities and $u \in \Omega_{W}(\bar{x})$ that there exists $\left(\beta_{1}, \beta_{2}\right) \in[-1,1] \times$ $[-1,1]$ satisfying

$$
\left\langle\alpha_{1}\left(2 u_{1}, 2 u_{2}+2\right)+\alpha_{2}\left(\beta_{1}, \beta_{2}\right), \bar{x}-u\right\rangle=-\left\langle\sum_{t \in T} \lambda_{t}^{g}(-t, 0)-\lambda_{1}^{H}(1,1)+\lambda_{1}^{G}(1,0), \bar{x}-u\right\rangle
$$




$$
\geq\left(\sum_{t \in T} \lambda_{t} g_{t}(u)-\sum_{i \in I_{l}} \lambda_{i}^{H} H_{i}(u)+\sum_{i \in I_{l}} \lambda_{i}^{G} G_{i}(u)\right)
$$

Reasoning by contraposition, suppose that $\left(\bar{x}, \bar{\alpha}, \bar{\lambda}^{g}, \bar{\lambda}_{1}^{G}, \bar{\lambda}_{1}^{H}\right)$ is not a weakly efficient solution of $\left(\mathrm{D}_{W}(\bar{x})\right)$. Then there exist $\left(u, \alpha, \lambda^{g}, \lambda^{G}, \lambda^{H}\right) \in \Omega_{W}(\bar{x})$ such that

$$
L\left(\bar{x}, \bar{\alpha}, \bar{\lambda}^{g}, \bar{\lambda}_{1}^{G}, \bar{\lambda}_{1}^{H}\right) \prec L\left(u, \alpha, \lambda^{g}, \lambda_{1}^{G}, \lambda_{1}^{H}\right) .
$$

Utilizing this along with $f(\bar{x})=L\left(\bar{x}, \bar{\alpha}, \bar{\lambda}^{g}, \bar{\lambda}_{1}^{G}, \bar{\lambda}_{1}^{H}\right), \alpha \in \mathbb{R}_{+}^{2}$ and $\sum_{i=1}^{2} \alpha_{i}=1$ gives us that $\left\langle\alpha, f(\bar{x})-L\left(u, \alpha, \lambda^{g}, \lambda^{G}, \lambda^{H}\right)\right\rangle<0$, which is equivalent to say that

$$
\sum_{i=1}^{2} \alpha_{i}\left(f_{i}(\bar{x})-f_{i}(u)\right)-\left(\sum_{t \in T} \lambda_{t} g_{t}(u)-\sum_{i \in I_{l}} \lambda_{i}^{H} H_{i}(u)+\sum_{i \in I_{l}} \lambda_{i}^{G} G_{i}(u)\right)<0 .
$$

The above relation together with (4.18) derives that

$$
\sum_{i=1}^{2} \alpha_{i}\left(f_{i}(\bar{x})-f_{i}(u)\right)<\left\langle\alpha_{1}\left(2 u_{1}, 2 u_{2}+2\right)+\alpha_{2}\left(\beta_{1}, \beta_{2}\right), \bar{x}-u\right\rangle,\left(\beta_{1}, \beta_{2}\right) \in[-1,1] \times[-1,1] .
$$

On the other hand, since $f_{1}, f_{2}$ are $\partial^{C}$-convexity at $u$ on $\mathbb{R}^{2}$, one has

$$
\begin{gathered}
\left\langle\left(2 u_{1}, 2 u_{2}+2\right), \bar{x}-u\right\rangle \leq f_{1}(\bar{x})-f_{1}(u), \\
\left\langle\left(\beta_{1}, \beta_{2}\right), \bar{x}-u\right\rangle \leq f_{2}(\bar{x})-f_{2}(u),
\end{gathered}
$$

which, taking into account $\alpha \in \mathbb{R}_{+}^{m}$, justifies that

$$
\left\langle\alpha_{1}\left(2 u_{1}, 2 u_{2}+2\right)+\alpha_{2}\left(\beta_{1}, \beta_{2}\right), \bar{x}-u\right\rangle \leq \sum_{i=1}^{2} \alpha_{i}\left(f_{i}(\bar{x})-f_{i}(u)\right) .
$$

This contradicts (4.19).

\section{Acknowledgments}

This work was supported by The Ministry of Education and Training of Vietnam under Grant No. B2022-TCT-01.

\section{REFERENCES}

[1] W. Achtziger, C. Kanzow, Mathematical programs with vanishing constraints: optimality conditions and constraint qualifications, Math. Program. 114 (2007), 69-99.

[2] T. Hoheisel, C. Kanzow, First- and second-order optimality conditions for mathematical programs with vanishing constraints, Appl. Math. 52 (2007), 495-514.

[3] T. Hoheisel, C. Kanzow, Stationarity conditions for mathematical programs with vanishing constraints using weak constraint qualifications, J. Math. Anal. Appl. 337 (2008), 292-310.

[4] T. Hoheisel, C. Kanzow, On the Abadie and Guignard constraint qualifications for mathematical programs with vanishing constraints, Optimization 58 (2009), 431-448.

[5] D. Dorsch, V. Shikhman, O. Stein, Mathematical programs with vanishing constraints: critical point theory, J. Glob. Optim. 52 (2012), 591-605.

[6] S.K. Mishra, V. Singh, V. Laha, R.N. Mohapatra, On constraint qualifications for multiobjective optimization problems with vanishing constraints. In: H. Xu, S. Wang, S.Y. Wu (eds.) Optimization Methods, Theory and Applications, pp. 95-135, Springer, Berlin, 2015. 
[7] S. Kazemi, N. Kanzi, Constraint qualifications and stationary conditions for mathematical programming with non-differentiable vanishing constraints, J. Optim. Theory Appl. 179 (2018), 800-819.

[8] A. Sadeghieh, N. Kanzi, G. Caristi, D. Barilla, On stationarity for nonsmooth multiobjective problems with vanishing constraints. J. Glob. Optim. doi: 10.1007/s10898-021-01030-1.

[9] S.K. Mishra, V. Singh, V. Laha, On duality for mathematical programs with vanishing constraints, Ann. Oper. Res. 243 (2016), 249-272.

[10] Q. Hu, J. Wang, Y. Chen, New dualities for mathematical programs with vanishing constraints, Ann. Oper. Res. 287 (2020), 233-255.

[11] A. Kabgani, M. Soleimani-damaneh, Characterization of (weakly/properly/robust) efficient solutions in nonsmooth semi-infinite multiobjective optimization using convexificators, Optimization 67 (2017), 217-235.

[12] N. Kanzi, S. Nobakhtian, Optimality conditions for nonsmooth semi-infinite multiobjective programming, Optim. Lett. 8 (2014), 1517-1528.

[13] N. Kanzi, On strong KKT optimality conditions for multiobjective semi-infinite programming problems with Lipschitzian data, Optim. Lett. 9 (2015), 1121-1129.

[14] O.I. Kostyukova, T.V. Tchemisova, Optimality conditions for convex semi-infinite programming problems with finitely representable compact index sets, J. Optim. Theory Appl. 175 (2017), 76-103.

[15] B. Mordukhovich, T.T.A. Nghia, Constraint qualifications and optimality conditions for nonconvex semiinfinite and infinite programs, Math. Program. 139 (2013), 271-300.

[16] B.S. Mordukhovich, Variational Analysis and Applications, Springer, New York, 2018.

[17] O. Stein, G. Still, Solving semi-infinite optimization problems with interior point techniques. SIAM J. Control Optim. 42 (2003), 769-788.

[18] L.T. Tung, Optimality conditions and duality for E-differentiable semi-infinite programming with multiple interval-valued objective functions under generalized E-convexity, J. Nonlinear Funct. Anal. 2020 (2020), Article ID 21.

[19] L.T. Tung, Strong Karush-Kuhn-Tucker optimality conditions for Borwein properly efficient solutions of multiobjective semi-infinite programming, Bull. Braz. Math. Soc. New Ser. 52 (2021), 1-22.

[20] L.T. Tung, H.D. Tam, Homeomorphic optimality conditions and duality for semi-infinite programming on smooth manifolds, J. Nonlinear Funct. Anal. 2021 (2021), Article ID 18.

[21] S.M. Guu, Y. Singh, S.K. Mishra, On strong KKT type sufficient optimality conditions for multiobjective semi-infinite programming problems with vanishing constraints, J. Inequal. Appl. 2017 (2017), 1-9.

[22] T. Antczak, Optimality conditions and Mond-Weir duality for a class of differentiable semi-infinite multiobjective programming problems with vanishing constraints, 4OR, doi: 10.1007/s10288-021-00482-1.

[23] L.T. Tung, Karush-Kuhn-Tucker optimality conditions and duality for multiobjective semi-infinite programming with vanishing constraints, Ann. Oper. Res. 2020. doi: 10.1007/s10479-020-03742-1

[24] L.T. Tung, Karush-Kuhn-Tucker optimality conditions and duality for semi-infinite programming problems with vanishing constraints, J. Nonlinear Var. Anal. 4 (2020), 319-336.

[25] J.P. Aubin, H. Frankowska, Set-Valued Analysis, Birkhäuser, Boston, 1990.

[26] A.A. Khan, C. Tammer, C. Zănilescu, Set-Valued Optimization, Springer-Verlag, Berlin, 2016.

[27] F. H. Clarke,Optimization and Nonsmooth Analysis, Wiley Interscience, New York, 1983.

[28] D.T. Luc, Theory of Vector Optimization, Springer, Berlin, 1989.

[29] A. Bagirov, N. Karmitsa, M.M. Mäkelä, Introduction to Nonsmooth Optimization: theory, practice and software, Springer, New York, 2014.

[30] T.W. Reiland, A geometric approach to nonsmooth optimization with sample applications. Nonlinear Anal. 11 (1987), 1169-1184.

[31] R.T. Rockafellar, Convex Analysis, Princeton Math. Ser., vol. 28, Princeton University Press, Princeton, New Jersey, 1970.

[32] M.A. Goberna, M.A. López, Linear Semi-Infinite Optimization, Wiley, Chichester, 1998.

[33] J.B. Hiriart-Urruty, C. Lemaréchal, Convex Analysis and Minimization Algorithms I, Springer, Berlin, 1993.

[34] P. Wolfe, A duality theorem for nonlinear programming, Q. Appl. Math. 19 (1961), 239-244.

[35] B. Mond, T. Weir, Generalized concavity and duality. In: S. Schaible, W.T. Ziemba (eds.) Generalized Concavity in Optimization and Economics, pp. 263-279, Academic Press, New York, 1981. 